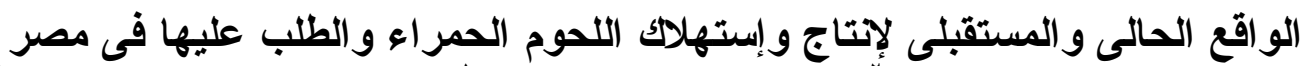

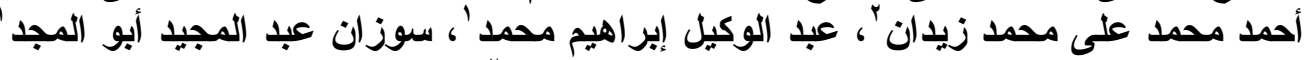

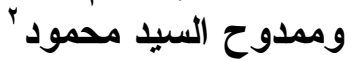
'قسم الاقتصاد الزراعى - كلية الزراعة - جامعة أسيوط. ' معهد بحوث الإقتصاد الزراعى - مركز البحوث الزعاعة الزعية - وزارة الزراعة.

Received on: 24/4/2016 Accepted for publication on: 10/5/2016

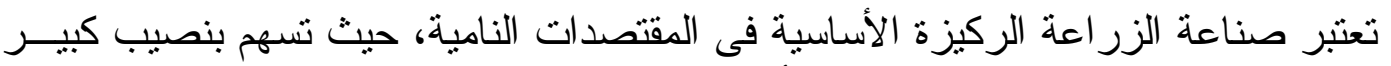

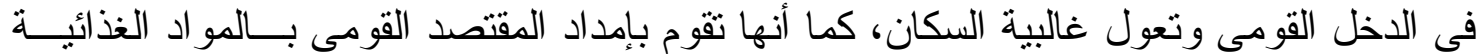

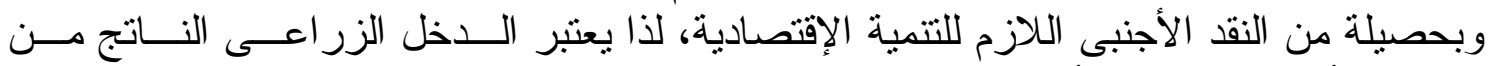

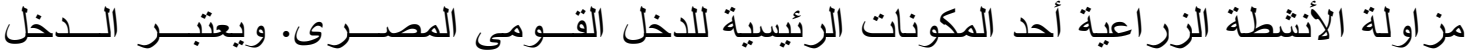

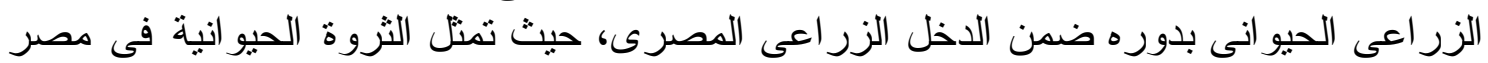

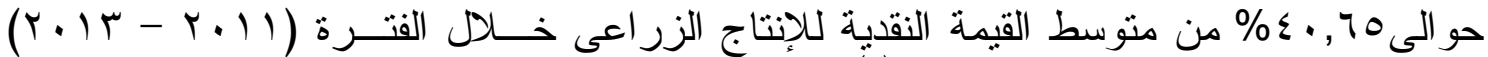

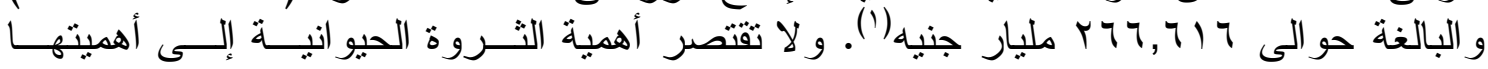

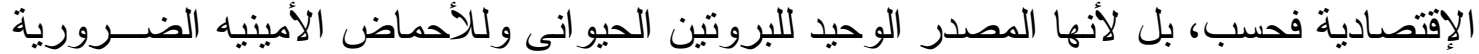

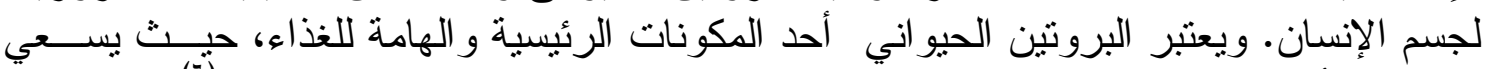

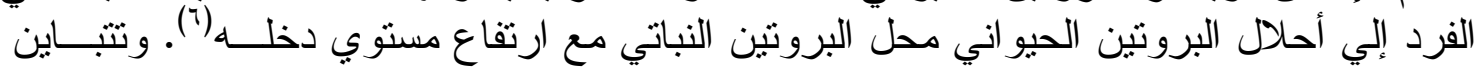

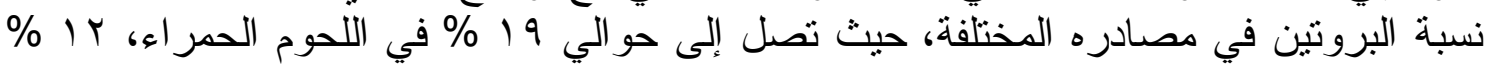

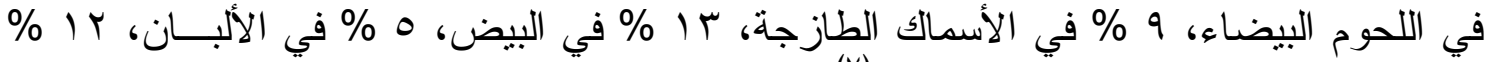

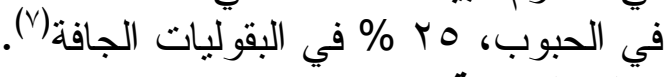
هذف الار اسة ابة

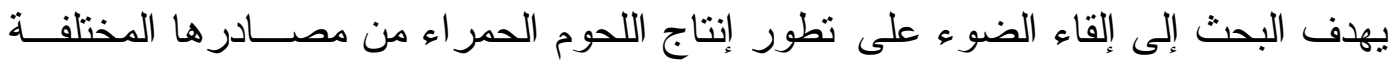

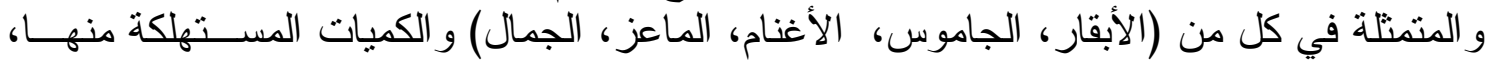

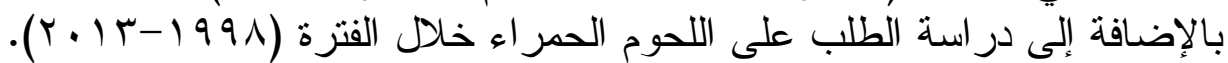

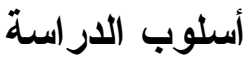

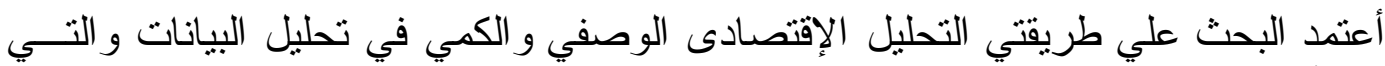

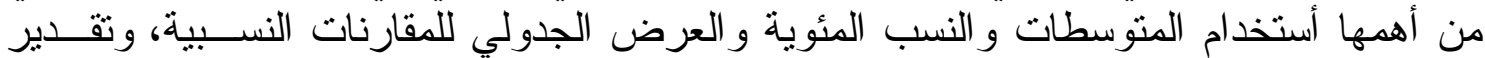

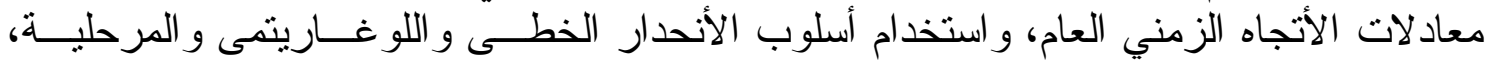

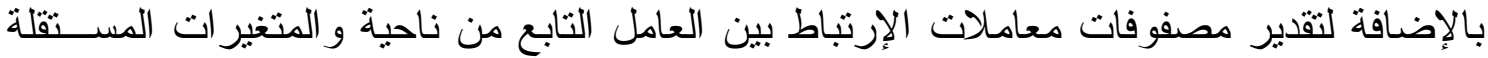

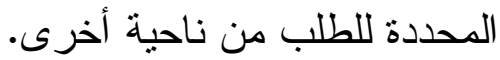

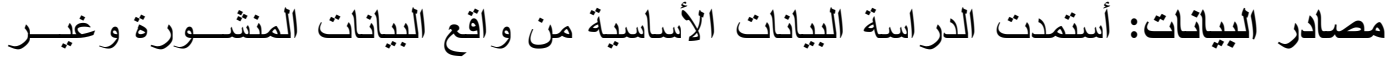

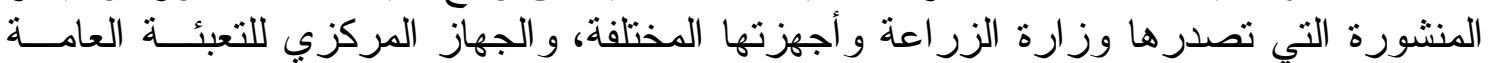
و الإحصاء، وبعض المر اجع و الأبحاث و الرسائل العلمية ذات الصلة العات بموضو ع البحث.

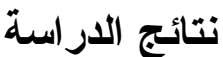

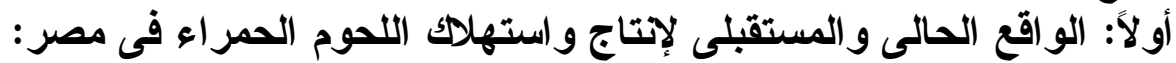

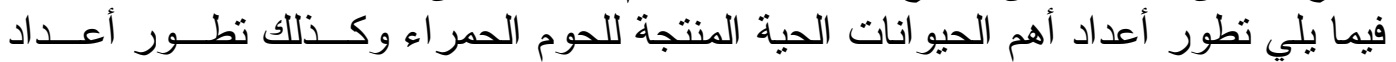

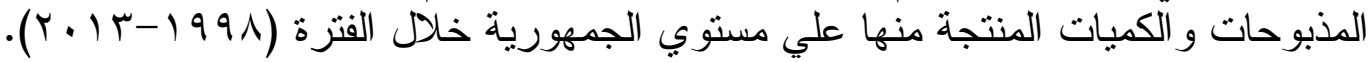

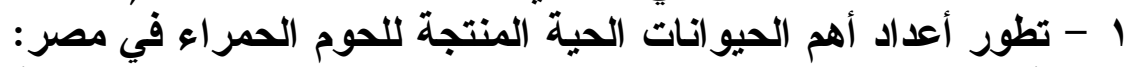

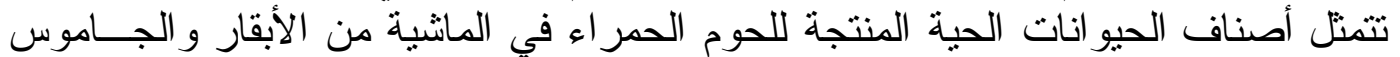

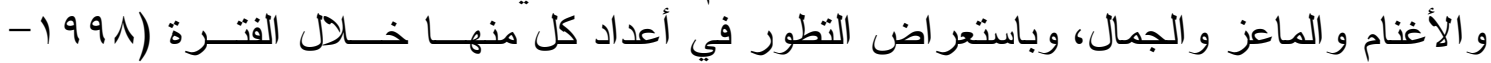

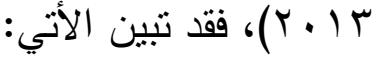


بدر اسة تطور أعداد الأبقار و الجاموس و الأغنام و الماعز و الجمال علي مستوي الجمهورية

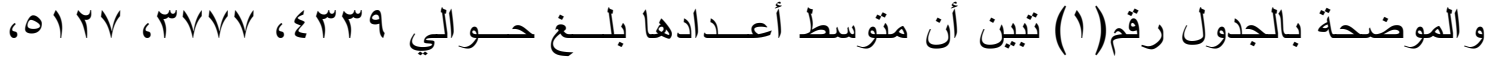

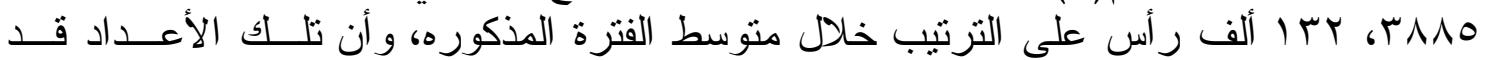
تز ايدت خلال هذه الفترة. وبتقدير معادلات الأتجاه الزمني العام لأعـــداد الماشــية و الحيو انــات

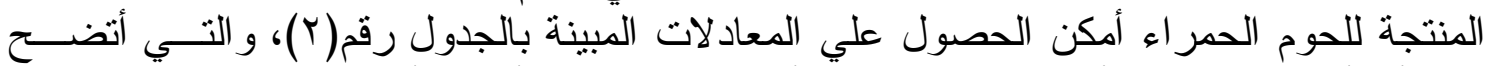
منها أن أعداد كل من الأبقار و الجاموس والأغنام و الماعز قد أخذت أتجاهاً عاماً متز ايداً معنوي

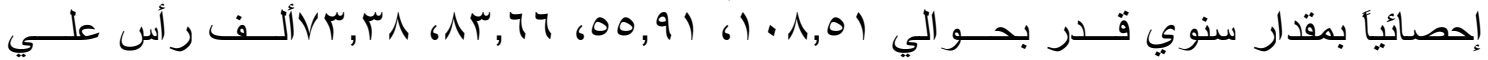

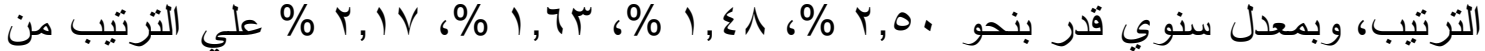

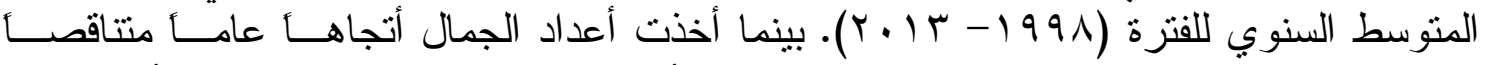

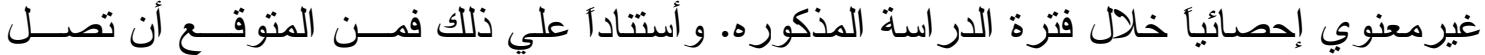

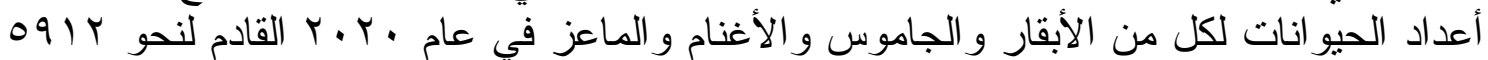

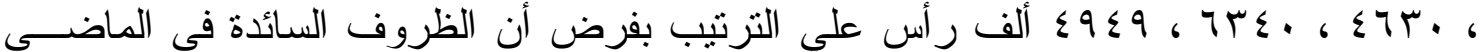
سوف تستمر فى المستقبل.

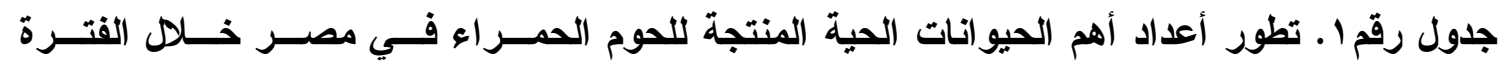
$\cdot(r \cdot 1 r-199 \Lambda)$

\begin{tabular}{|c|c|c|c|c|c|c|c|c|c|c|}
\hline \multicolumn{2}{|c|}{ الجمال } & \multicolumn{2}{|c|}{ الماعز } & \multicolumn{2}{|c|}{ الأغنام } & \multicolumn{2}{|c|}{ الجاموس } & \multicolumn{2}{|c|}{ الأبقار } & \multirow[b]{2}{*}{ السنة } \\
\hline $\begin{array}{c}\text { معدل) النمو(\%) } \\
\end{array}$ & (ألف رأس) & النمو(\%) معد) & 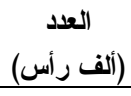 & النمو(\%) معد) & (ألف رأس) & النمو (\%عل) & 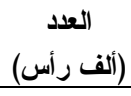 & النمو(\%) معد) & \begin{tabular}{|c} 
(ألف رأس) \\
(أسد)
\end{tabular} & \\
\hline- & $1 \leqslant r$ & - & Trדו & - & ETOr & - & $M) \leqslant q$ & - & rrIV & 1991 \\
\hline$(0,7 \pi)$ & $1 \pi \varepsilon$ & $1, \leqslant \varepsilon$ & A.rT & $\cdot, 9$. & $\{r q \mid$ & 0,10 & . & $7, Y_{0}$ & $r \leqslant 1 \wedge$ & 1999 \\
\hline$(\cdot, \vee \cdot)$ & $1 \leqslant 1$ & $0, \cdot r$ & $r \leq r o$ & $r, 79$ & $\leq \leqslant 79$ & $\vee, r \cdot$ & rrvq & $9, \vee r$ & ror. & $r \ldots$ \\
\hline$(0,7 \pi)$ & $1 \pi \varepsilon$ & $V, r \varepsilon$ & $r \leqslant 9 V$ & V,rT & $\leq 7 V 1$ & 17,19 & איסט & 11,10 & rA. & $r \ldots l$ \\
\hline$(1 \cdot, 07)$ & IrV & $9, \wedge \leqslant$ & rodr & IV,r. & 01.0 & $1 \wedge, \cdot \varepsilon$ & TVIV & $r \neg, \wedge q$ & $\varepsilon \cdot \lambda r$ & $r \ldots r$ \\
\hline$(\Sigma, r T)$ & ד4 & $17, \wedge V$ & rᄉा & $1 T, \leq 9$ & 纟q५q & $19,9 \varepsilon$ & TVVV & $r_{1, \varepsilon .}$ & $E Y T V$ & $r \ldots r$ \\
\hline$(9,10)$ & 149 & 11,90 & rᄉv & $10, \wedge 1$ & $0 . \leqslant r$ & $r, 1$. & $\Gamma \wedge \leqslant 0$ & ro,v^ & 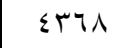 & $r \ldots \varepsilon$ \\
\hline$\cdot, \cdots$ & $1 \leqslant r$ & $17,7 r$ & rᄉ.r & $r \cdot, r r$ & OYTY & rT, TV & 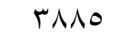 & $r q, \varepsilon r$ & $\leqslant \leqslant \wedge 0$ & $r \ldots$ \\
\hline$\varepsilon, Y T$ & $1 \leqslant 1$ & $1 \wedge, \wedge 9$ & rAVV & $r r, V \varepsilon$ & orso & $r o, \cdot r$ & rquv & $\varepsilon r, \Gamma$. & (4). & $r \ldots r$ \\
\hline$(\varepsilon \cdot, \wedge 0)$ & $\Lambda \varepsilon$ & $r q, 1 r$ & हा। & ro,Tr & $0 \leqslant 7 V$ & $r \cdot, r q$ & $\leqslant 1.0$ & or, r & हqur & $r \ldots v$ \\
\hline$(Y \leqslant, 70)$ & $1 \cdot v$ & $r v, I V$ & $\varepsilon \varepsilon V \mu$ & ru,ru & $0 \leqslant 91$ & $\langle\wedge, \vee\rangle$ & $\varepsilon .0 r$ & $07,1 \leq$ & $0 . r \mu$ & $r \ldots \Lambda$ \\
\hline$(r, O r)$ & ITV & $r 7,9 r$ & हाएव & $r \wedge, \leq 0$ & 009. & $(1,91$ & r人rq & $\varepsilon \cdot, 77$ & soro & $r \ldots q$ \\
\hline$(r), \wedge r)$ & 111 & $r \wedge, \cdot r$ & \&IVo & $r V, \cdot V$ & oor. & Y),$Y \leq$ & ґᄉו & $\varepsilon \vee, \ldots$ & EVYq & $r \cdot 1$. \\
\hline$(r, O r)$ & ITV & $r \cdot, o v$ & $\sum r O \Lambda$ & $r r, Y \wedge$ & 0 oryo & $Y \nearrow, 乏 \wedge$ & rqAr & $\sum \wedge, 09$ & $\leq \vee \wedge$. & $r .11$ \\
\hline$\cdot, \cdots$ & $1 \leqslant r$ & $r, .0$ & $\varepsilon r \cdot \tau$ & $r \varepsilon, V V$ & $0 \leqslant T$. & $r, r_{T}$ & $\leqslant 170$ & or,vo & $\leq 9 \leq 7$ & $r .1 r$ \\
\hline $\mathrm{V}, \mathrm{VO}$ & 104 & rV, ro & Elor & $r v, \wedge 0$ & $007 \varepsilon$ & $r \varepsilon, \Gamma r$ & ralo & $\varepsilon \vee, 0$. & $\leq V \leq 0$ & $r+1 r$ \\
\hline & Irr & & 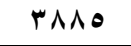 & & $01 Y V$ & & rvv & & $\leqslant r r q$ & المتوسط \\
\hline
\end{tabular}

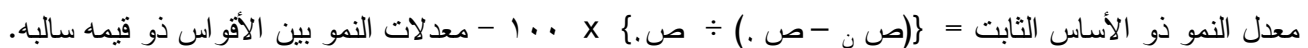

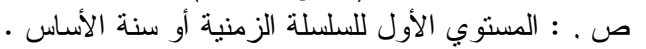

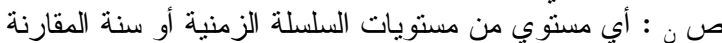
المصــدر : جمعت وحسبت من بيانات الجهاز المركزي للتعبئة العامة و الإحصـاء، نشرة إحصـــــاءات الثـروة الحيو انيــة

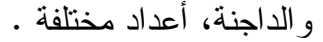


جدول رقم ب. معادلات الأتجاه الزمني العام لأعداد الحيوانات الحية المنتجة للحوم الحمراء في مصــر خـلهل

\begin{tabular}{|c|c|c|c|c|c|c|c|}
\hline \multirow{3}{*}{ 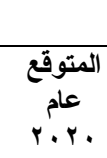 } & \multirow{2}{*}{\multicolumn{2}{|c|}{ متوسط التغير }} & \multirow[b]{3}{*}{ الظتوسط } & \multirow[b]{3}{*}{ r } & \multirow[b]{3}{*}{ ف } & \multicolumn{2}{|c|}{ 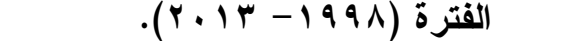 } \\
\hline & & & & & & & \\
\hline & $\begin{array}{c}\text { المئوى } \\
\text { (\%) }\end{array}$ & (ألف رأس) & & & & المعادلة & البيان \\
\hline $091 \mathrm{r}$ & $r, 0$. & $* * 1 \cdot \wedge, 01$ & $\varepsilon r r q$ & $\cdot, A \cdot V$ & 01,07 & 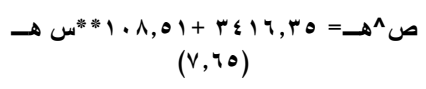 & الأبقار \\
\hline$\sum \pi r$. & l, ¿^ & $* * 00,91$ & TrYV & $\cdot, v \cdots$ & $r T, T V$ & 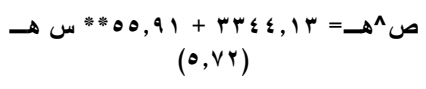 & 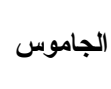 \\
\hline . & $1, \pi$ & ***人ґ, ४ & $01 Y V$ & $\cdot, \wedge \uparrow \wedge$ & $T V, 01$ & 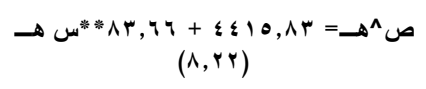 & الأغنام \\
\hline$\varepsilon q \leqslant 9$ & r,IV & $* * \vee r, r \wedge$ & riso & $\cdot, \wedge \varepsilon 1$ & $V \Psi, q \varepsilon$ & 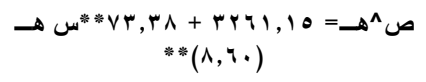 & الماعز \\
\hline- & $(\cdot, r \cdot)$ & $(\cdot, r Y \varepsilon)$ & ITr & $\cdot, \cdots 4$ & $\cdot, \cdot \vee \vee 9$ & 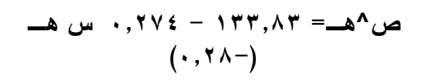 & الجمال \\
\hline
\end{tabular}

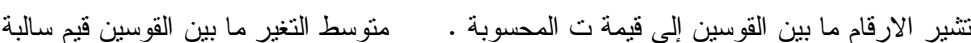

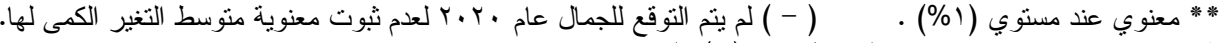
المصدر : حسبت من بيانات الجدول رقم( (1) السابق.

r - تطور أعداد المذبوحات لأهم الحيواتات المنتجة للحوم الحمر اء في مصر :

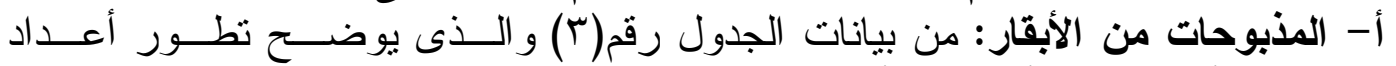

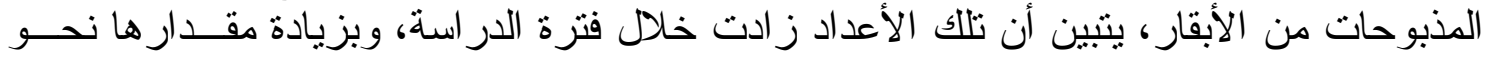

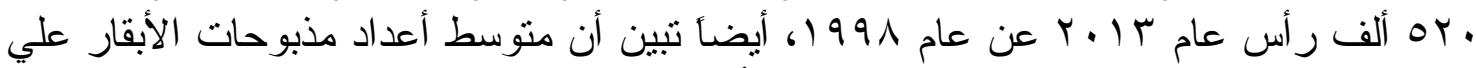

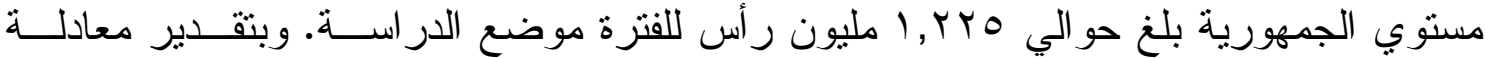

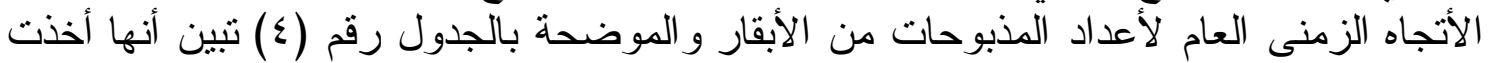

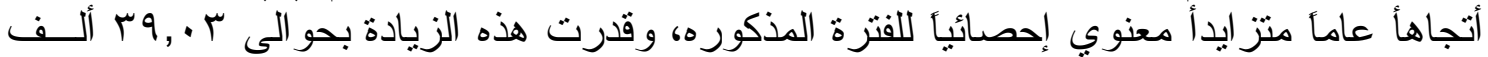

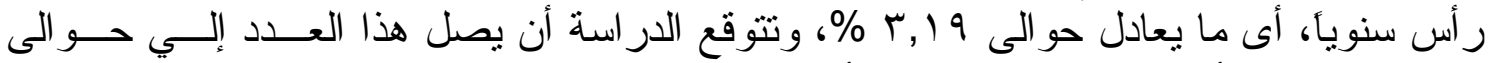

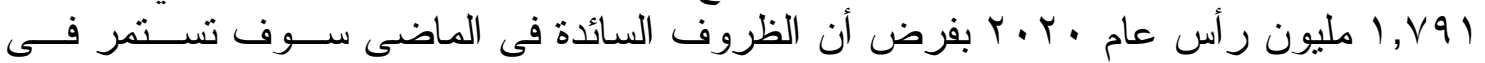
المستقبل.

ب- المذبوحات من الجاموس: بالنسبة لأعداد مذبوحات الجاموس و الموضحة بالجـــدول

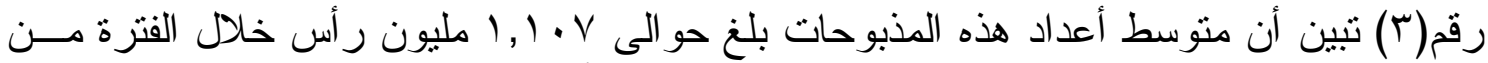

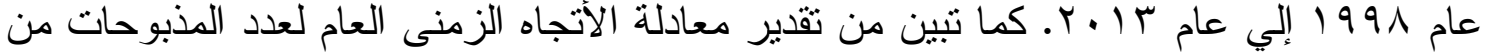

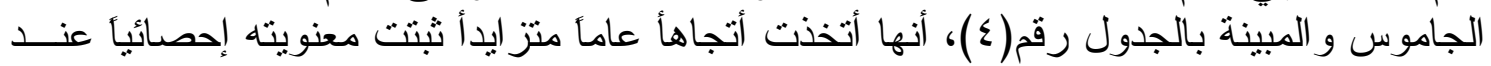

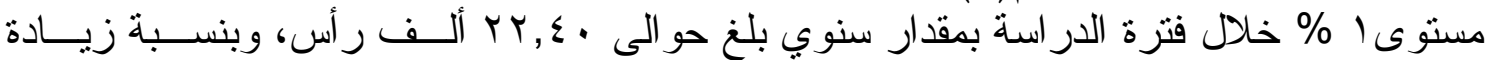

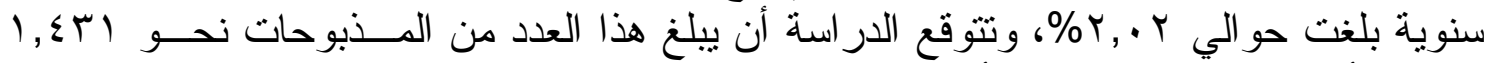

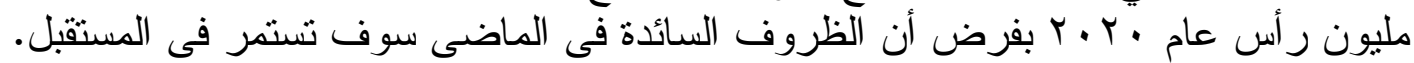

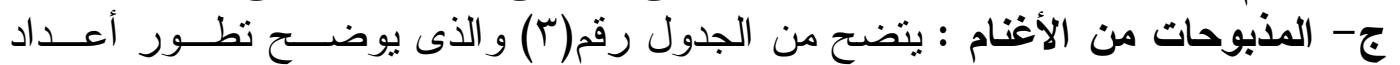

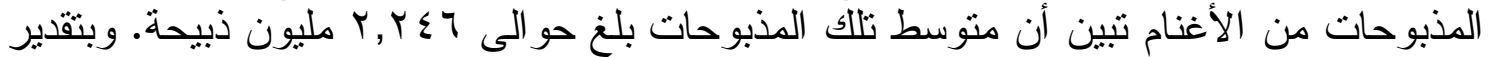

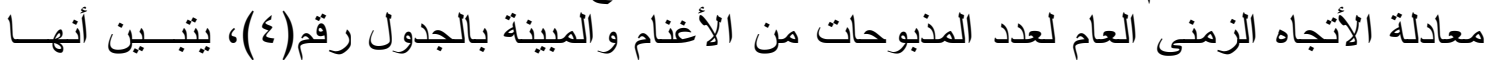

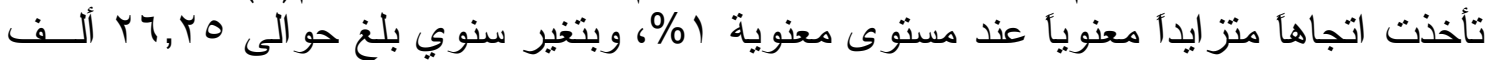

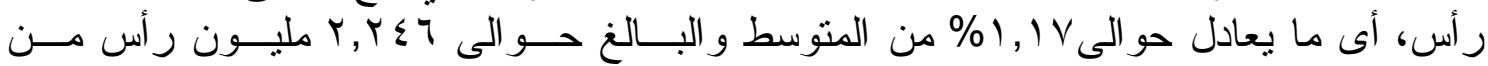

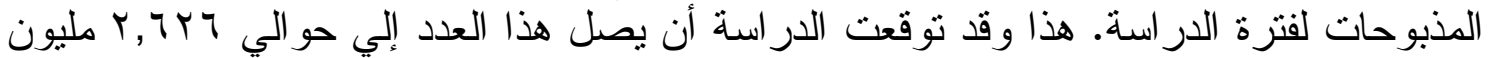

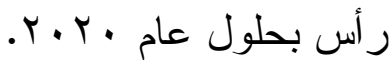




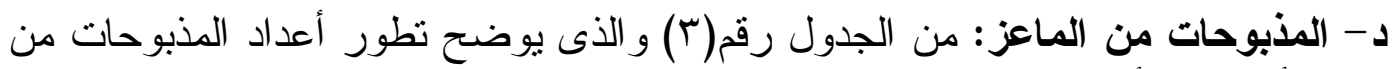

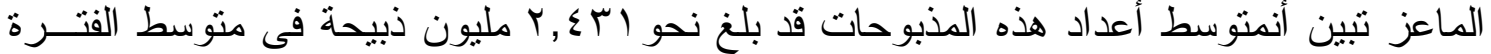

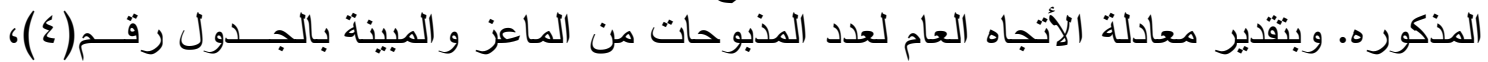

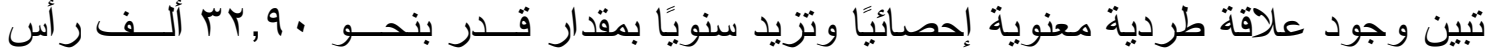

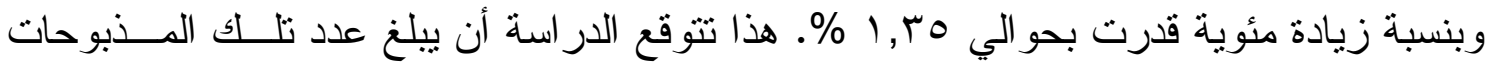

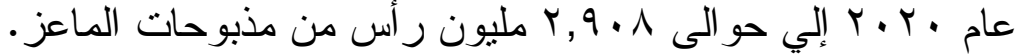

جدول رقمّا. تطور أعداد المذبوحات من أهم الحيوانات المزرعية المنتجة للحوم الحمراء في مصــر

\begin{tabular}{|c|c|c|c|c|c|c|c|c|c|c|}
\hline \multicolumn{2}{|c|}{ الجمال } & \multicolumn{2}{|c|}{ الماعز } & \multicolumn{2}{|c|}{ الأغنام } & \multicolumn{2}{|c|}{ الجاموس } & \multicolumn{2}{|c|}{ الأبقار } & \multirow[b]{2}{*}{ السنة } \\
\hline النمو (\% م) & (ألف رأس) & $\begin{array}{c}\text { النمو(\%) } \\
\text { (\% }\end{array}$ & (ألف رأس) & النمو(\%) معل & (ألف رأس) & النمو(\%) معل & (ألف رأس) & $\begin{array}{c}\text { النمو (\%) } \\
\text { (\%) }\end{array}$ & (ألف رأس) & \\
\hline- & ro & - & rוrq & - & $19 N Y$ & - & 940 & - & $1 \wedge 9$ & 1991 \\
\hline$(0, \times 1)$ & Tr & $\cdot, \wedge \varepsilon$ & riov & $(1,+7)$ & 1971 & $1, r q$ & $9 \leq 1$ & $\Lambda, 77$ & 977 & 1999 \\
\hline$\cdot, \cdots$ & ro & $r, q r$ & אTYT & $1,9 r^{\prime}$ & $r \cdot r$. & 1,94 & 904 & $11, r 0$ & $9 \wedge 9$ & $r \ldots$ \\
\hline$(0, \times)$ & אי & $0,9 \leq$ & TYTA & $\varepsilon, .9$ & Tr. & r, & 977 & $11,9 r$ & 990 & $r \ldots l$ \\
\hline$(\wedge, \circ \vee)^{\prime}$ & rt & $\wedge, 9 \wedge$ & וזrז & 10,19 & TrQO & IT,VT & $1.0 \leqslant$ & $r \wedge, \wedge$. & $11 \leq 0$ & $r \ldots r$ \\
\hline$(\curlyvee, \wedge ૫)$ & $r \varepsilon$ & $I \varepsilon, V V$ & $r \leqslant 00$ & 17,10 & $r M \cdot r$ & $1 r, .9$ & $1 \cdot \leqslant \Lambda$ & rq, ro & $11 \leq 9$ & $r . . r$ \\
\hline$(\wedge, \circ \vee)^{\prime}$ & rt & $1 \leqslant, 0 \leqslant$ & $r \leq 0$. & $11, \leqslant 0$ & rr. $q$ & $1 \leq, r Y$ & 1.71 & & $11 \wedge \mathrm{V}$ & $r \ldots \varepsilon$ \\
\hline$r, \wedge \tau$ & דו & $1 \cdot, 19$ & rTOV & 10,99 & rrqq & $19, r_{0}$ & 1110 & ru,vA & $1 Y 17$ & $r \ldots$ \\
\hline $0, \times 1$ & rV & r & . דצr. & $1 \wedge, \leqslant \vee$ & $r T \leqslant \Lambda$ & $r_{1}, \cdot V$ & ITr & $\{r, \varepsilon 1$ & 1 1Y77 & $r \ldots r$ \\
\hline$(\varepsilon \cdot, \cdots)$ & rI & $r, \wedge \Lambda$ & $r T \cdot V$ & $19, Y Y$ & אדצי & $r \varepsilon, \wedge)$ & 1178 & $\sum r, O r$ & IYTV & $r . . v$ \\
\hline$(Y Y, \wedge T)$ & TV & $r Y, V V$ & TYTY & 19,71 & TrVT & $r \tau, \varepsilon r$ & $111 \mathrm{r}$ & or, r & Iros & $r . . \wedge$ \\
\hline$\cdot, \cdots$ & ro & $|\wedge, 7|$ & rorv & $1 \wedge, r V$ & $r T \leq T$ & r) & I TrA & $0 \wedge, 9 \leq$ & $1 \leqslant 14$ & $r . . q$ \\
\hline$(r \cdot, \cdots)$ & rᄉ & $r \cdot, r \leq$ & TOVY & $\backslash \wedge, \vee V$ & TrOS & $r \leq, 9 V$ & IKTY & & $1 \leq 0 \leq$ & $r \cdot 1$. \\
\hline$\cdot, \cdots$ & ro & YY,YO & YIIO & $17, \Gamma$. & rT.O & $r_{0, q} \varepsilon$ & 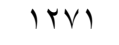 & $7 r, .9$ & $1 \leq \varepsilon 1$ & $r .11$ \\
\hline$\cdot, \cdots$ & ro & $r \varepsilon, 0$. & אדצ & $|V, \wedge|$ & סTrT & $r \wedge, \wedge \wedge$ & $1 \% .0$ & $7 \varepsilon, \leqslant 0$ & $1 \leqslant 7 r$ & $r .1 r$ \\
\hline$\Lambda, O V$ & rᄉ & 11,07 & rory & $r \cdot r r$ & r TrT & $r_{0,1}$ & $11 \mathrm{~V}$. & $O \wedge, \leqslant q$ & $1 \leq .9$ & $r .1 r$ \\
\hline & $r r$ & & $r \leq r \mid$ & & $r r \leq q$ & & 11.8 & & IYYO & المتوسط \\
\hline
\end{tabular}

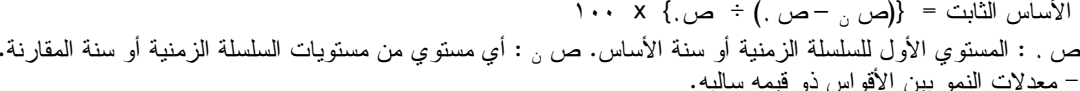

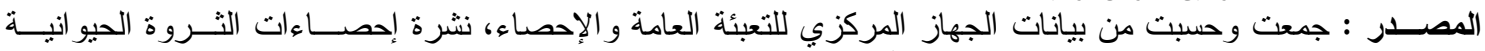

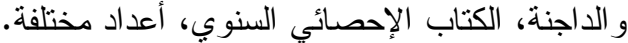

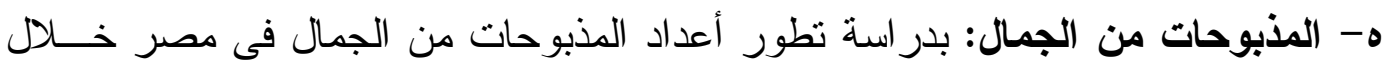

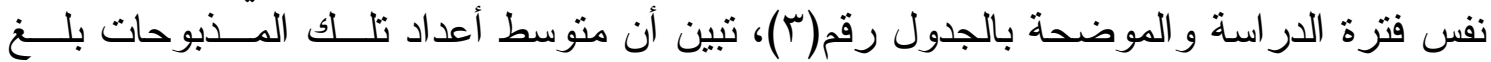
حو الى rr ألف ر أس. وبتقدير معادلة الأتجاه العام لعدد المذبوحات من الجمال و المبينة بالجدول

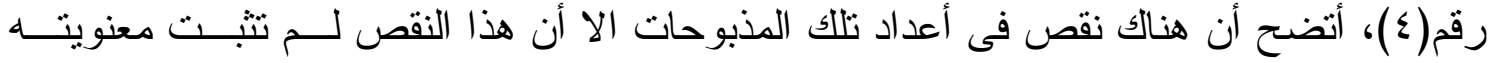
إحصائيًا.

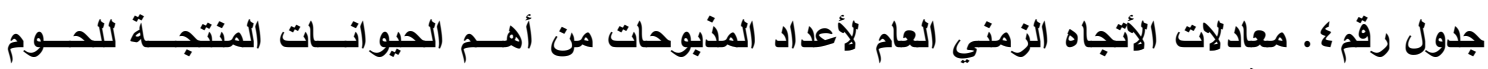

\begin{tabular}{|c|c|c|c|c|c|c|c|}
\hline \multirow{2}{*}{ 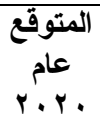 } & \multicolumn{2}{|c|}{ متوسط التغير } & \multirow{2}{*}{ الظاهرة } & \multirow[b]{2}{*}{ ru } & \multirow[b]{2}{*}{ ف } & \multirow[b]{2}{*}{ المعادلة } & \multirow[b]{2}{*}{ البيان } \\
\hline & $\begin{array}{c}\text { المئوى } \\
\text { (\%) }\end{array}$ & (ألف رأسى) & & & & & \\
\hline$|v 9|$ & $r .19$ & $* * r q, \cdot r$ & Irro & $\cdot, 9 \leqslant V$ & rol,0V & 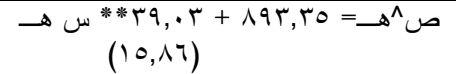 & الأبقار \\
\hline $1 \leqslant \pi$ & $r, \cdot r$ & ***r, & $11 . \mathrm{V}$ & $\cdot, 9 \vee \pi$ & $97, \varepsilon r$ & 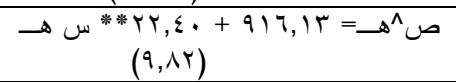 & الجاموس \\
\hline דצY & $1,1 \mathrm{~V}$ & ***Y, & $r T \leq T$ & $\cdot, 790$ & MI,QY & 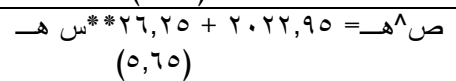 & الأغنام \\
\hline$r q .1$ & 1, ro & $* * r r, q$. & $r \leqslant r 1$ & $\cdot, \wedge 17$ & 71,90 & 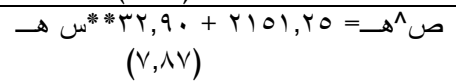 & الماعز \\
\hline
\end{tabular}




\begin{tabular}{|c|c|c|c|c|c|c|c|}
\hline- & $(\cdot, \cdot 9)$ & $(\cdot, \cdot r)$ & rr & $\cdot, \cdots)$ & $\cdot,+10$ & 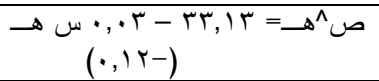 & الجمال \\
\hline
\end{tabular}

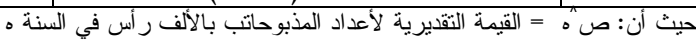

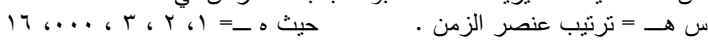

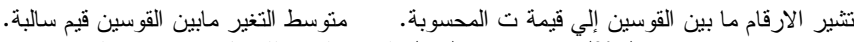

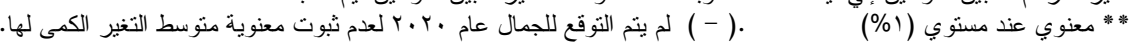

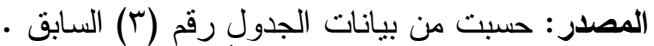

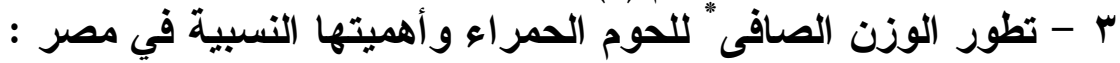

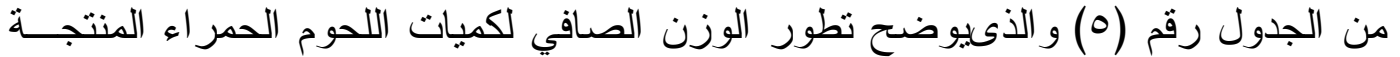

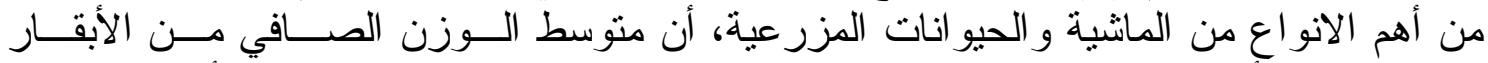

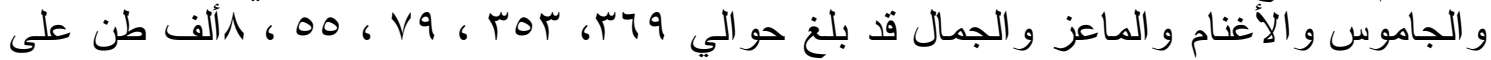

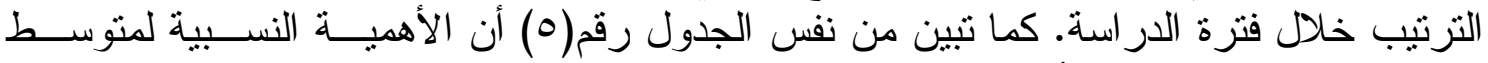

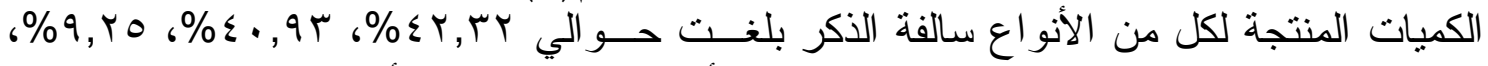

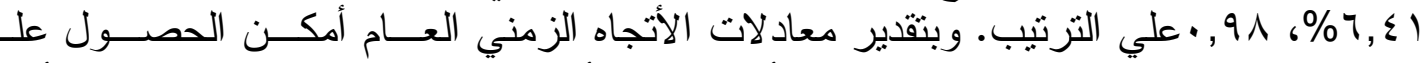

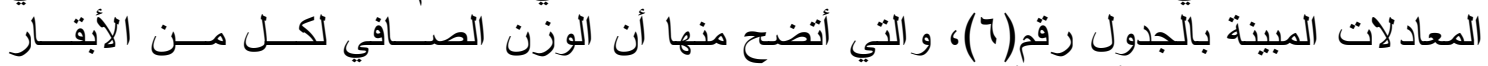

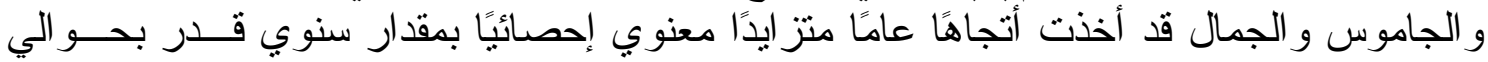

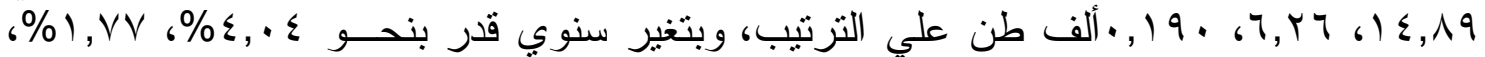

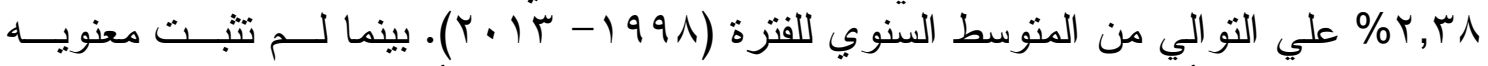

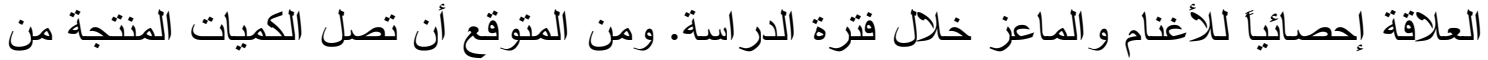

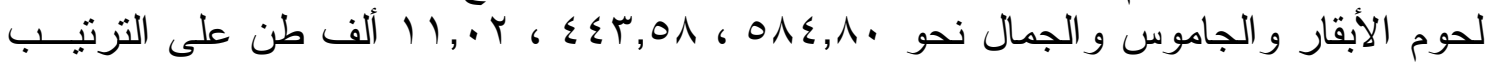

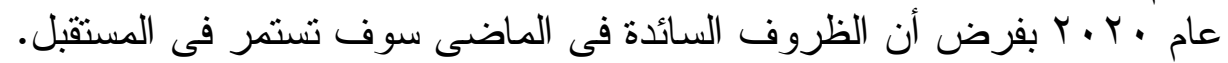

جدول رقمه. تطور الوزن الصافي لكميات اللحوم الحمراء المنتجة من أهم الأنواع في مصـر خــله

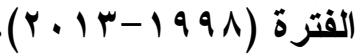

\begin{tabular}{|c|c|c|c|c|c|c|c|c|c|c|c|c|c|c|c|}
\hline \multicolumn{3}{|c|}{ الجمال } & \multicolumn{3}{|c|}{ الماعز } & \multicolumn{3}{|c|}{ الأغتام } & \multicolumn{3}{|c|}{ الجاموس } & \multicolumn{3}{|c|}{ الأبقار } & \multirow[b]{2}{*}{ البيان } \\
\hline |الأهمية & النمو & (ألفزن & 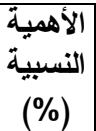 & 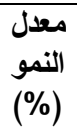 & |لوزن & الأهمبية & 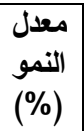 & (الوزن & الأهمبية & 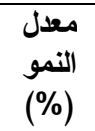 & |الوزن & الأهمبية & النمو & |الوزن & \\
\hline $1, .4$ & - & $\Lambda$ & $\neg, \varepsilon \mathrm{V}$ & - & o. & 9,11 & - & $\overline{v 1}$ & $\$ 9,00$ & . & rAT & rr, & . & $r \circ \lambda$ & 1991 \\
\hline 1,14 & $\cdot, \ldots$ & $\wedge$ & $V, r \leqslant$ & $\cdot, \cdots$ & o. & $1 \cdot, r V$ & $\cdot,$. & vi & $\{1, r q$ & $(Y \otimes, r r)$ & ץАT & $r 9,01$ & $0, \Lambda_{1}$ & rVr & 1999 \\
\hline $1,1 \pi$ & $\cdot, \ldots$ & $\wedge$ & $V, r T$ & $r, \ldots$ & 01 & 1., & $r, A r$ & $v r$ & $\llbracket \cdot, \wedge 0$ & $(r \leqslant, \Lambda \cdot)$ & $r \wedge \Lambda$ & $\varepsilon,$, & $9, r$. & rAY & r... \\
\hline 1,10 & $\cdot, \ldots$ & $\wedge$ & $\vee, \leqslant \wedge$ & $\varepsilon, \ldots$ & or & $1 \cdot, \vee \vee q$ & $0,4 \pi$ & vo & $\varepsilon \cdot, \wedge \uparrow$ & $(r \circ, \wedge 0)$ & $r \wedge \varepsilon$ & rq, rA & $0, \wedge 1$ & rVT & $r \ldots r$ \\
\hline$\cdot, \Lambda_{0}$ & $(1, Y, 0)$. & v & $\curlyvee, 01$ & $\wedge, \ldots$ & 0 \& & $1 ., 11$ & $18,9$. & $\Lambda r$ & $\varepsilon 1, r q$ & $(11, \leqslant 9)$ & rrq & \&, व & $r \cdot, r r$ & צדי & $r \ldots r$ \\
\hline $1, \ldots$ & $\cdot, \ldots$ & $\wedge$ & $v, .9$ & $1 \leqslant, \ldots$ & ov & $1 \cdot, r$. & $10, \leqslant 9$ & Ar & $\varepsilon \cdot, r$. & $(10, \varepsilon \cdot)$ & rY & \&, , qr & rV,Or & rrq & $r \ldots r$ \\
\hline $1,1$. & IY,O. & 9 & $V, r$. & $1 \wedge, \ldots$ & 09 & $1 \cdot,{ }^{1}$ & $|\Lambda, r|$ & $\wedge \varepsilon$ & צ & $(1+, 0)$ & זיז & $\varepsilon r, V \varepsilon$ & צו & ro. & $r \ldots \varepsilon$ \\
\hline - , $9 \leqslant$ & $\cdot, \ldots$ & $\wedge$ & $\uparrow, \varepsilon$ & $1 \cdot, \ldots$ & 00 & $9, \vee 1$ & $18,9$. & גז & «1, «. & $(V, \diamond V)$ & Y & $\left\{1, r^{\prime}\right.$ & $r ४, \wedge r$ & ror & $r \ldots o$ \\
\hline $1, . r$ & Ir,O. & 9 & צ & $1 \cdot, \ldots$ & 00 & $9,4 \wedge$ & $19, V Y$ & ^。 & $\{1, \ldots$ & $(7,+1)$ & ז. & $\varepsilon 1, \wedge$. & $\varepsilon Y, Y_{0}$ & rqv & $r \ldots r$ \\
\hline$\cdot, \infty 0$ & $(r v, 0 \cdot)$ & 。 & 7,70 & $r r, \ldots$ & ו & $9, r \wedge$ & $r, 1 r$ & 14 & $\varepsilon, r_{0}$ & $(r, r q)$ & rv. & \& & OY,Tr & rar & $r \ldots v$ \\
\hline זי, & $\left(r_{0}, \cdots\right)$ & 4 & $4, \leqslant 0$ & $r \varepsilon, \ldots$ & $4 r$ & $\wedge, 90$ & $r, 1 r$ & $\wedge 4$ & $r q, \cdot r$ & $(r, .9)$ & rvo & $\varepsilon \varepsilon, V_{0}$ & Vד, ציו & $\leqslant$ «. & $r \ldots \wedge$ \\
\hline Fr & $\cdot, \cdots$ & A & $4, .1$ & $1 \wedge, \ldots$ & 09 & ^, & $19, V Y$ & ^。 & $\Gamma \wedge, \wedge \varepsilon$ & $(\cdot, \Delta r)$ & ral & $\{0,0 \mathrm{~V}$ & VY,Y & « « V & $r \ldots q$ \\
\hline -,91 & Ir,o. & 9 & & $\uparrow, \ldots$ & r & $v, 04$ & $0,4 \pi$ & vo & 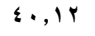 & $r, q r$ & ra1 & «४,•V & $V V, I T$ & \&OV & .1. \\
\hline 1,11 & $r v, o$. & 11 & $0, \pi 4$ & $\uparrow, \ldots$ & or & $v, \varepsilon q$ & $\varepsilon, r r$ & $v \varepsilon$ & $\{\cdot, \cdot \wedge$ & $r, r q$ & एव & $\{0,90$ & $v_{0}, q v$ & \& & 4.11 \\
\hline 1,11 & $r v, 0$. & 11 & $0, \leqslant 0$ & $\wedge, \cdots$ & 0 \& & $\vee, ० \wedge$ & $0,4 \pi$ & vo & 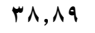 & •, Or & rıo & $\{५, 9 \vee$ & $\Lambda \cdot, r \mu$ & \& & $r \cdot 1 T$ \\
\hline $1, r \varepsilon$ & $0 ., \ldots$ & ir & $0, r q$ & $\varepsilon, \ldots$ & or & $\vee, \wedge \wedge$ & $\mathrm{v}, \cdot \varepsilon \varepsilon$ & v4 & $\varepsilon \cdot, r \mid$ & $1,0 \mathrm{~V}$ & rAq & $\varepsilon 0, \cdot \wedge$ & $\checkmark \wedge, \uparrow$ & \&ro & $r+1 r$ \\
\hline$\cdot, 9 \wedge$ & & $\Lambda$ & $7, \leqslant 1$ & & 00 & $9, r_{0}$ & & Vq & $\varepsilon \cdot, 94$ & & ror & $\varepsilon r, r r$ & & $r 99$ & لمتوسط \\
\hline
\end{tabular}

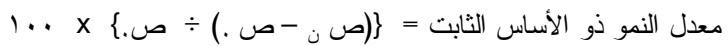

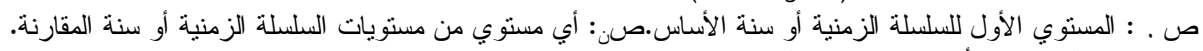
- معدلات النمو بين الأقو اس ذو قيمه سالبه.

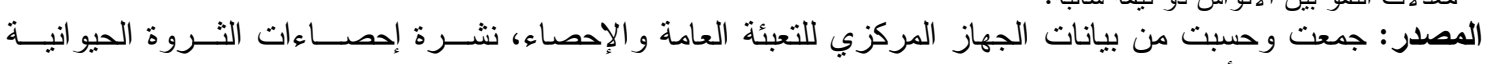
و الداجنة، أعداد مختلفة. 
جدول رقم \. معادلات الأتجاه الزمني العام للوزن الصافى للحوم الحمراء المنتجة من أهم الأنواع فــي

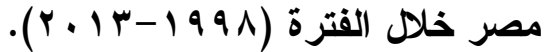

\begin{tabular}{|c|c|c|c|c|c|c|c|}
\hline \multirow{2}{*}{ المتوقع عام ب.r. } & \multicolumn{2}{|c|}{ متوسط التغير } & \multirow{2}{*}{ مت الظوسط } & \multirow[b]{2}{*}{ ז } & \multirow[b]{2}{*}{ ف } & \multirow[b]{2}{*}{ المعادلة } & \multirow[b]{2}{*}{ البيان } \\
\hline & $\begin{array}{l}\text { المئوى } \\
\text { (\%) }\end{array}$ & (ألف رأسي) & & & & & \\
\hline $0 \wedge \varepsilon, \wedge$. & $\varepsilon, \cdot \varepsilon$ & $* * 1 \leqslant, \wedge 9$ & r79 & • & $r \cdot r, r)$ & 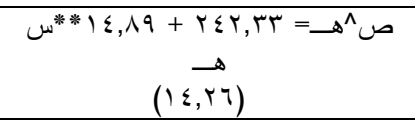 & الأبقار \\
\hline$\leq \varepsilon r, 01$ & $1, V V$ & $* * \Upsilon, Y \uparrow$ & ror & $\cdot, 07 \leqslant$ & $1 \wedge, 1 r$ & 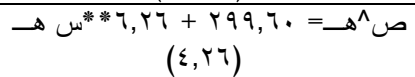 & الجاموس \\
\hline- & $\cdot, r \varepsilon$ & $\cdot, Y V$ & vq & $\cdot, \cdot \leq 9$ & $\cdot, V r$ & $\begin{array}{c}\rightarrow \omega^{* * *} \cdot, Y V+V I, V 0=\rightarrow^{\wedge} ص \\
(\cdot, \wedge 0)\end{array}$ & الأغنام \\
\hline - & $\cdot, \leqslant V$ & $\cdot, Y 4$ & 00 & $\cdot, 11$ & $1, \mathrm{~V}$ & $\begin{array}{c}\rightarrow \omega \cdot, Y Y+O Y, O \Lambda=\rightarrow^{\wedge} \text { ص } \\
(1, r Y)\end{array}$ & الماعز \\
\hline $11, \cdot r$ & r,ru & $* ., 19$ & $\wedge$ & $\cdot, r \leq V$ & $\varepsilon, 7$. & $\begin{array}{c}\rightarrow \omega^{*} \cdot, 19+7,70=\rightarrow^{\wedge} ص \\
(r, 10)\end{array}$ & الجمال \\
\hline
\end{tabular}

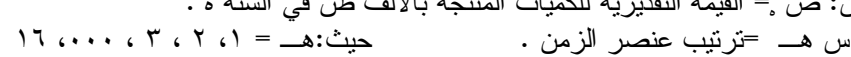

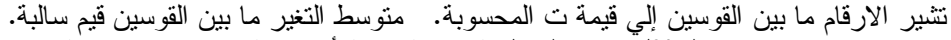

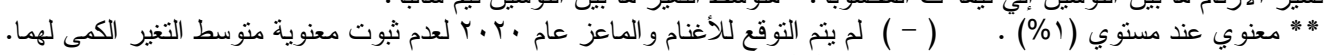

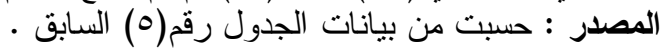

\section{تظور الكميات المستهلكة من اللحوم الحمراء :}

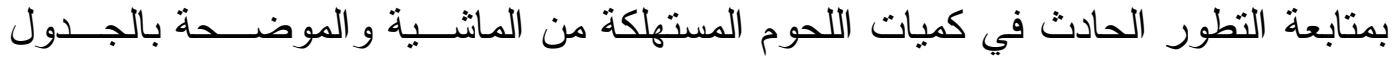

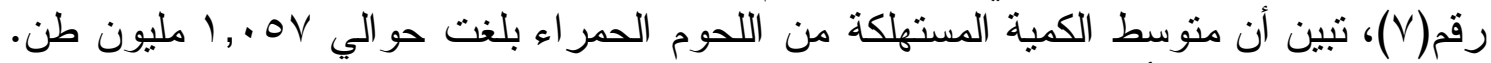

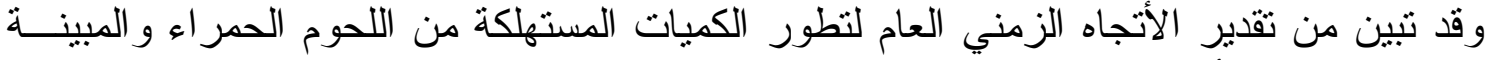

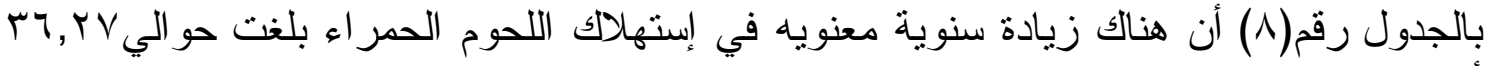

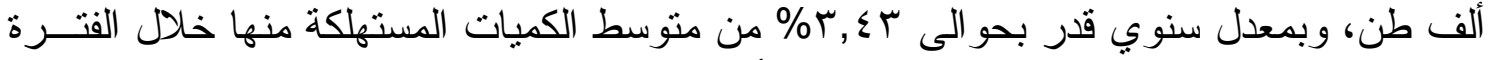

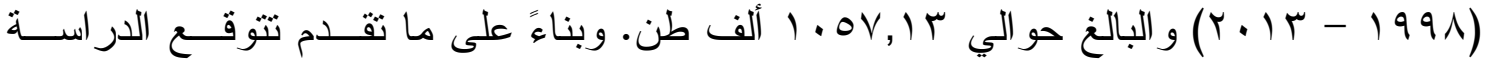

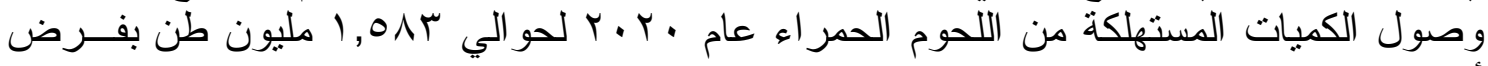
أن الظروف السائدة فى الماضى سوف تستمر في المستقبل.

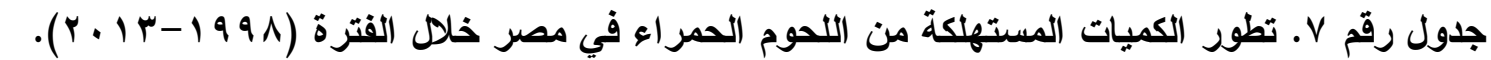

\begin{tabular}{|c|c|c|c|c|}
\hline معدل النمو & متوسط نصيب & معدل النمو & 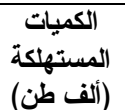 & السنة \\
\hline$(1, \cdots)$ & Ir,Ar & 1,10 & V9o & 1999 \\
\hline$\varepsilon, \vee q^{\prime}$ & Ir,ov & $q, Y q$ & 109 & $r \ldots$ \\
\hline$(0,1 V)$ & $M, Y A$ & $1, r$ & Vq & $r \ldots$ \\
\hline $7, V Y$ & $M, \wedge r$ & IA,r & qr. & $r . . r$ \\
\hline$\varepsilon, Y_{0}$ & $1 \pi, 0$. & $18,9 \leq$ & qrV & $r \ldots \varepsilon$ \\
\hline $17,1 \leq$ & $10, \cdot \varepsilon$ & & 1.04 & r..o \\
\hline$r v, \varepsilon q$ & 17,01 & $\varepsilon q, \wedge \vee$ & $11 \mathrm{VA}$ & $r \ldots T$ \\
\hline & $\mid v, 1$. & $0 \wedge, 70$ & $1 Y \leqslant V$ & $r \ldots v$ \\
\hline$q, r \leq$ & $1 \varepsilon, 17$ & $\leqslant 7,90$ & 1100 & $r \cdot 1 r$ \\
\hline \multirow[t]{2}{*}{$19, \mathrm{VV}$} & 10,01 & $70,1 \leq$ & 1491 & $r .1 T$ \\
\hline & $1 \leqslant, \vee 0$ & & $1.0 \mathrm{~V}, 1 \mathrm{r}$ & المتوسط \\
\hline
\end{tabular}

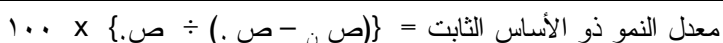

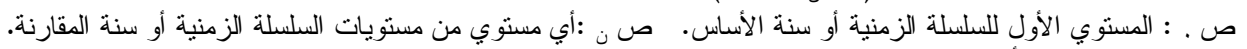
- معدلات النمو بين الأقو اس ذو قيمه سالبه. 
Assiut J. Agric. Sci., (47) No. (4) 2016 (251-262)

Website: http://www.aun.edu.eg/faculty_agriculture

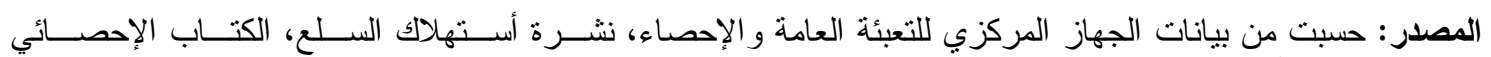

ISSN: 1110-0486

E-mail:ajas@aun.edu.eg 
جدول رقم^ء. معادلات الأتجاه الزمني العام للكميات المستهلكة ونصيب الفرد من اللحوم الحمراء فـى هـ

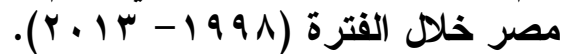

\begin{tabular}{|c|c|c|c|c|c|c|c|}
\hline \multirow{2}{*}{ 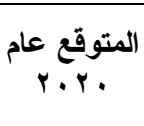 } & \multicolumn{2}{|c|}{ متوسط التغير } & \multirow{2}{*}{ الظوسط } & \multirow[b]{2}{*}{ Yנ } & \multirow[b]{2}{*}{ ف } & \multirow[b]{2}{*}{ المعادلة } & \multirow[b]{2}{*}{ البيان } \\
\hline & $\begin{array}{c}\text { المئوى) } \\
\text { (\%) }\end{array}$ & (ألف طمن) & & & & & \\
\hline 10人r,.. & $r, \varepsilon r$ & $* * \Gamma 4, Y V$ & $1.0 \mathrm{~V}, 1 \mathrm{r}$ & $\cdot, \wedge \backslash \wedge$ & $T r, \lambda T$ & 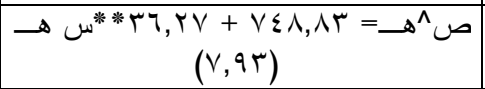 & كميات مستهلكة \\
\hline IV,VA & $1, \varepsilon r$ & $* * \cdot, r)$ & $1 \leqslant, V_{0}$ & $\cdot, \varepsilon \cdot \uparrow$ & 9,01 & $\begin{array}{c}\left.\rightarrow \omega^{* * *} \cdot, r\right)+1 r, 90=\rightarrow^{\wedge} ص \\
(r, 1 \cdot)\end{array}$ & نصيب الفرد \\
\hline
\end{tabular}

حيث:هـ

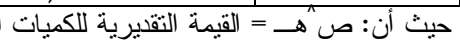
س هـ تنثير الارقام ما بين القوسين إلي قيمة ت ت المحسوبة .

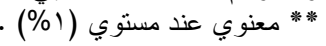
المصدر : حسبت من بيانات الجدول رقم(V) السابق .

تطور الإستهلاك الفردي من اللحوم الحمر اء:

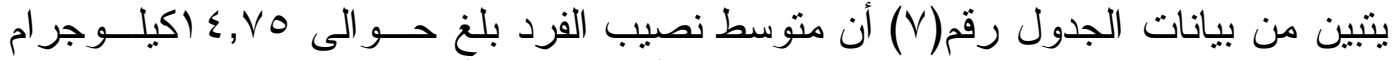

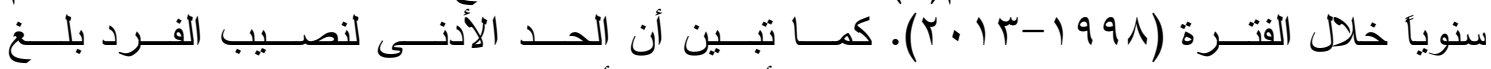

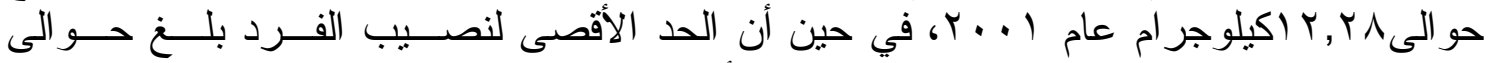

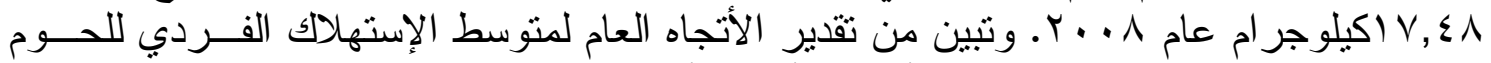

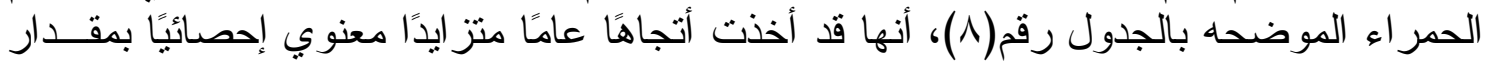

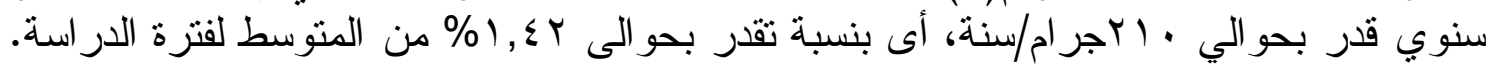

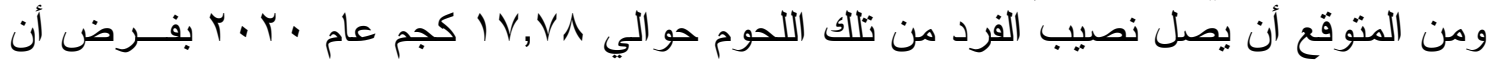

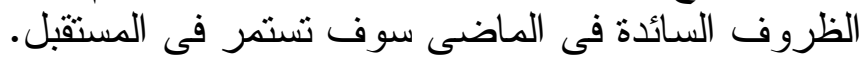

تقدير دالة الطلب على اللحوم الحمر اءع بإستخدام الأسعار الجارية:

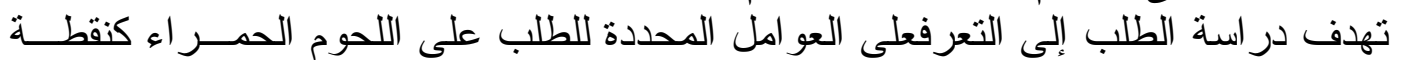

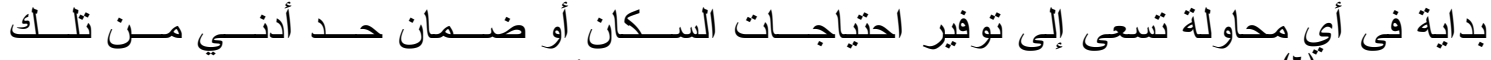

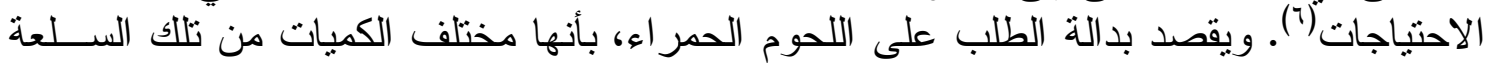
التى ير غب المستهلك فى الحصول عليها، نظير ثمن معين وفى سوق معينة وفى زمسـن معــين.

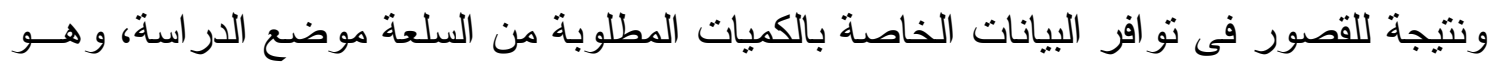

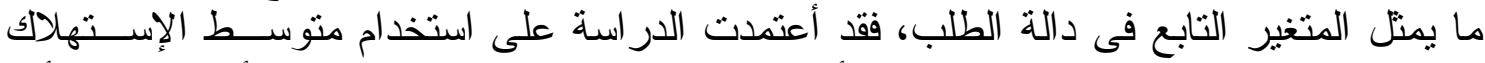

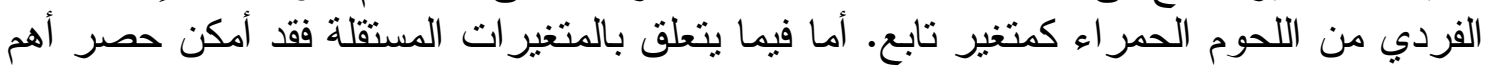

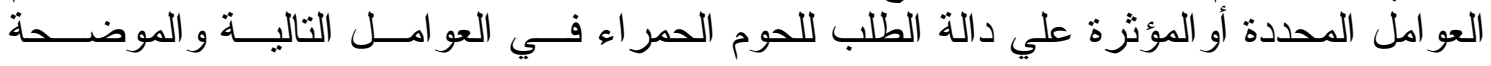
بالجدول رقم (9) كما يلي: ( (1) المتغير التابع " صد "وهي": وهو عبارة عن نصيب الفرد من اللحوم الحمر اء بــالكيلوجر ام

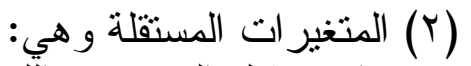

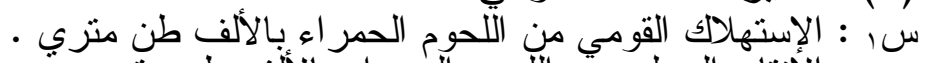

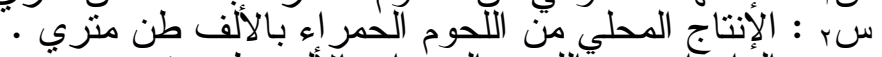

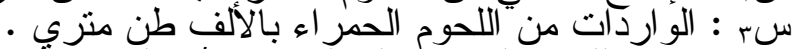

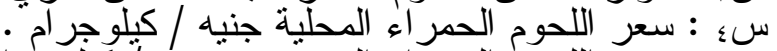

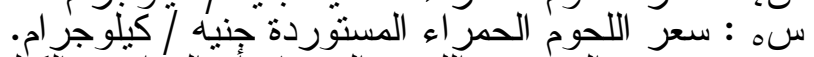

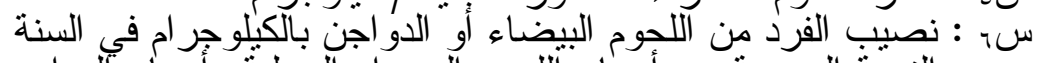

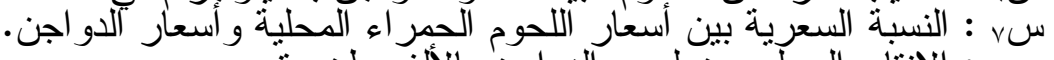

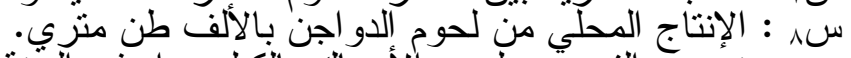

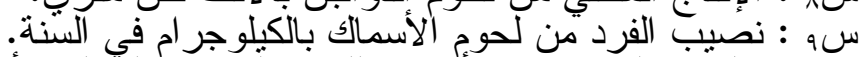

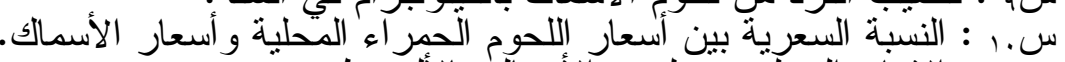
س1, : الإنتاج المحلي من لحوم الأسماك بالألف طن منري. 


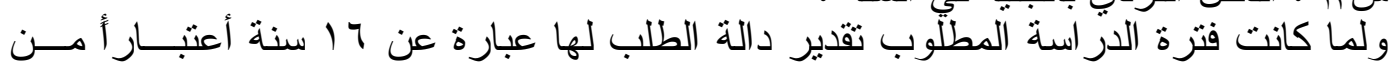

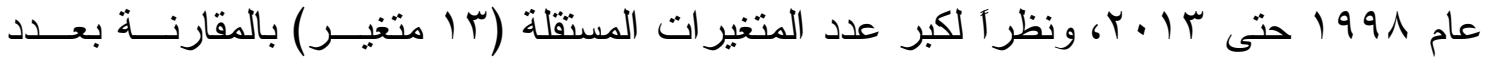

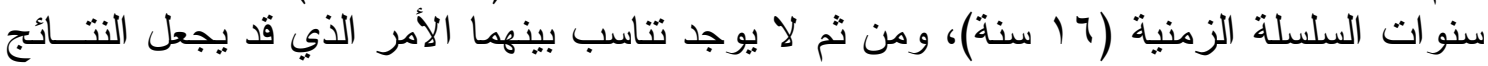

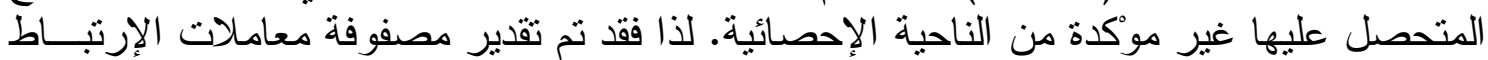

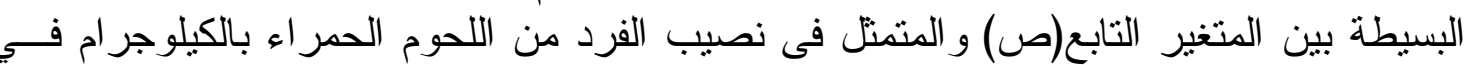

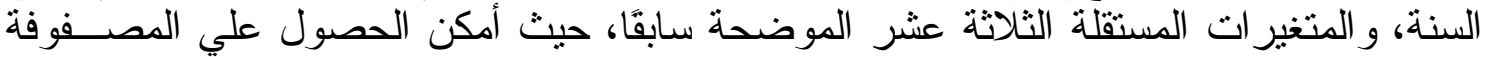

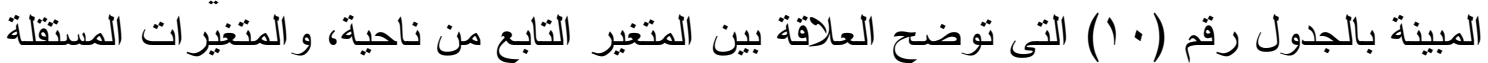

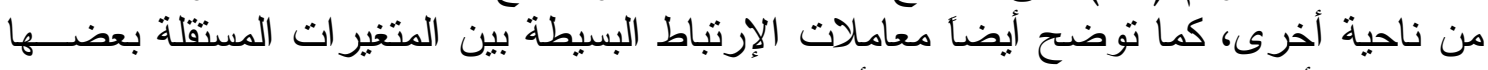

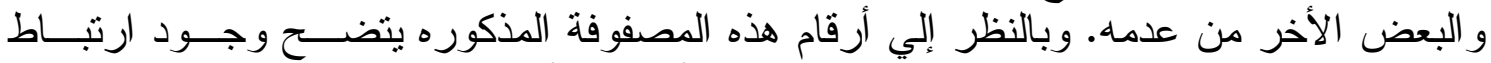

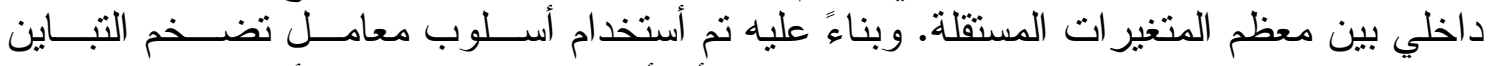
Variance Inflation Factor(VIF)

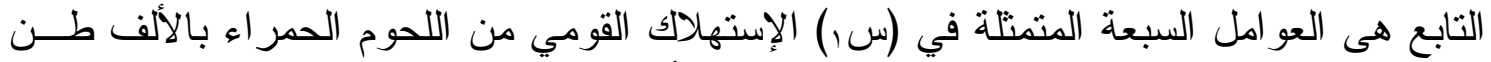

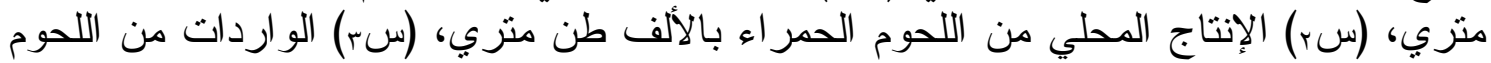

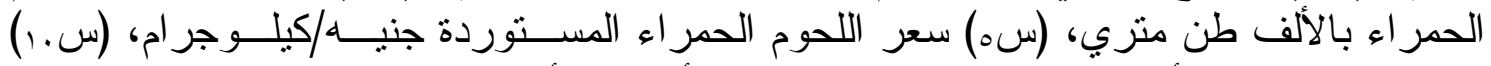

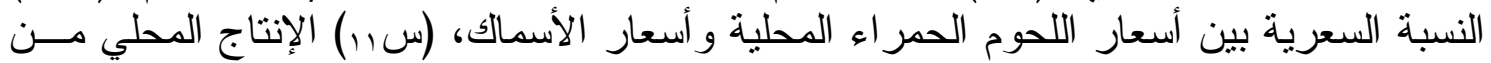

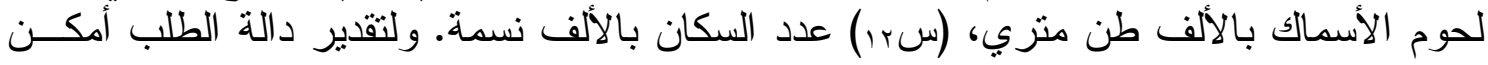

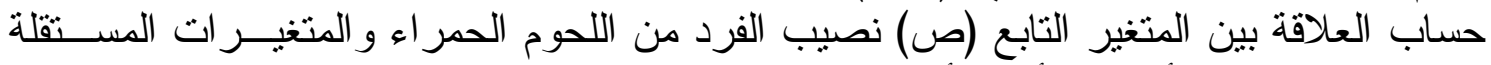

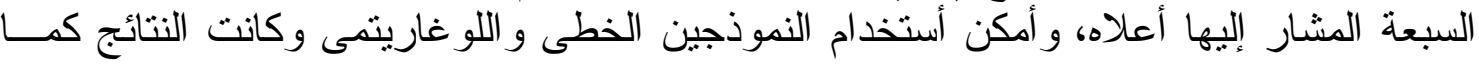

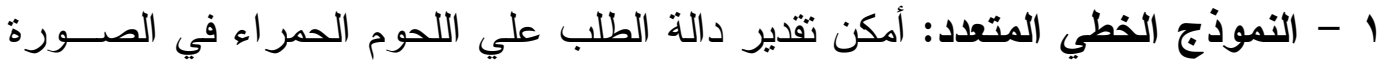

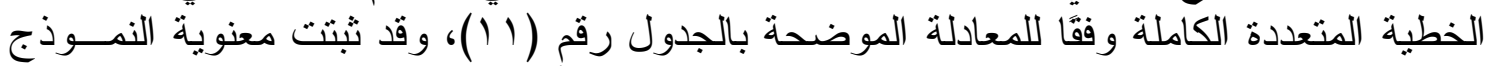

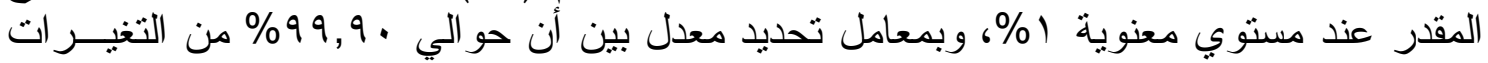
الحادثة فى منوسط نصيب الفرد من اللحوم الحمر اء ر اجعهللتغير ات فيى العو امل المستقلة ســالفة

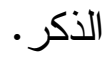

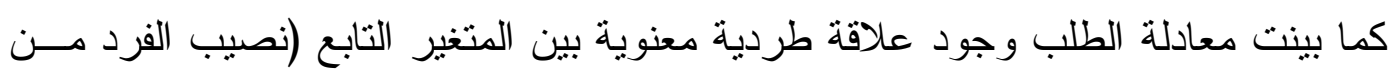

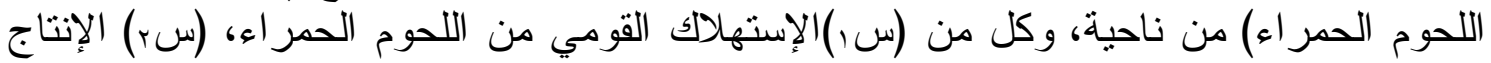

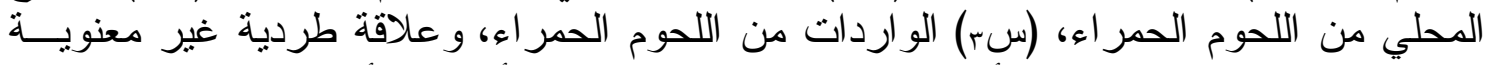

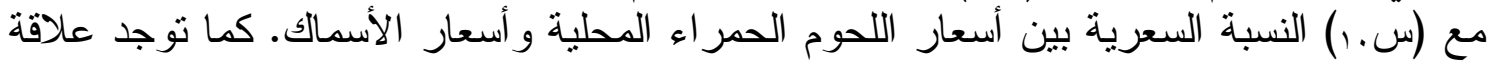

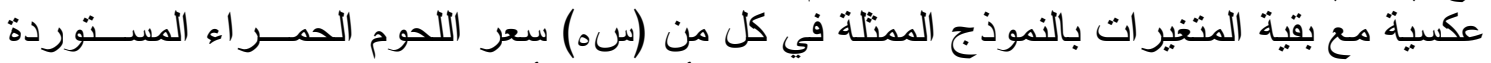

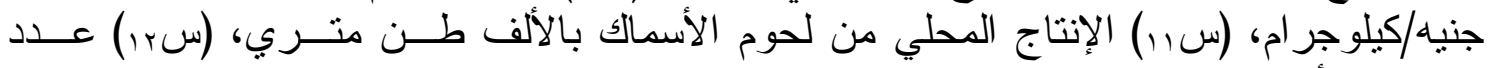

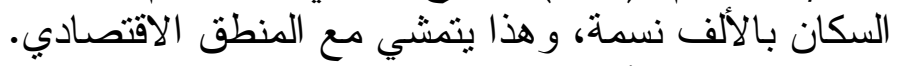

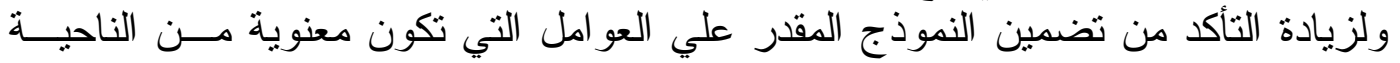

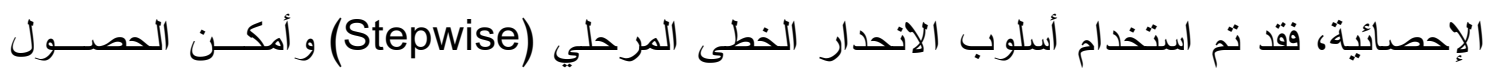

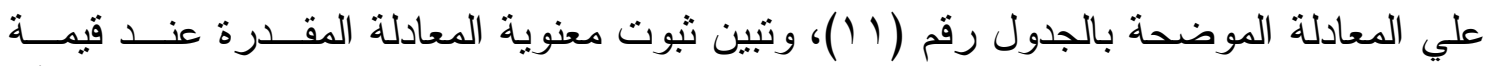

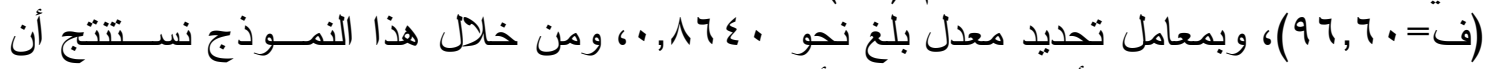

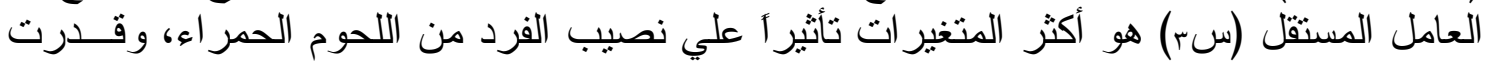

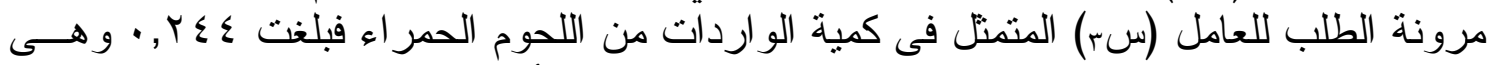

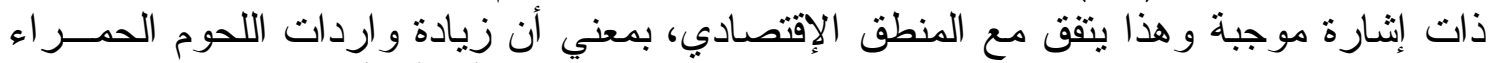

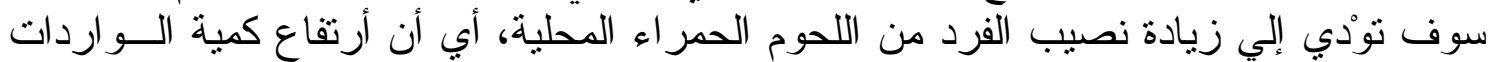




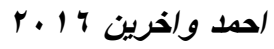

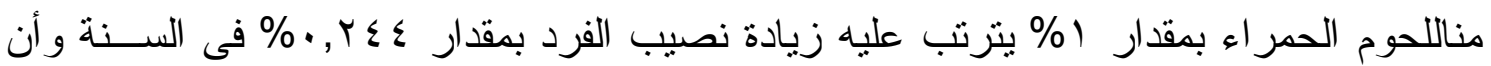
العلاقة التي نزبطهم هي علاقة طردية. 
جدول رقم 9.

\begin{tabular}{|c|c|c|c|c|c|c|c|c|c|c|c|c|c|c|}
\hline الإخلي & ألفكان & الألحلي ألأمن & السعرية اللسبة & الفرد منب الأبمانك & 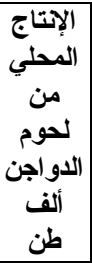 & السعربة اللحبة & 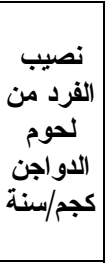 & الاستثير اد & 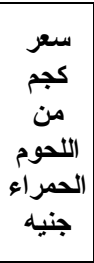 & 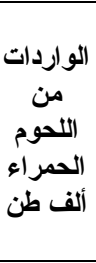 & الإلتاج & الاستهلاك & 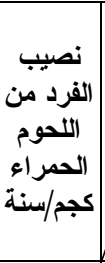 & |لبيان \\
\hline$\Sigma \curlyvee \wedge$. & 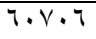 & OrT & $r, 10$ & 11,17 & 017 & $T, q \leq$ & $\wedge, 0$. & 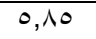 & $0,9$. & $11 \leqslant, \cdot$ & TVT,. & $\vee \wedge \neg, \cdot$ & 14,90 & 1.1 \\
\hline$\varepsilon 9.7$ & 71994 & q & Y,T & Ir, T. & Tr人 & $r, 7)$ & $1 \cdot, 11$ & $0,7 \leqslant$ & $17,7 \mathrm{~V}$ & 1.0, & $791, \cdot$ & $\vee 90,$. & $M, A r$ & 99 \\
\hline OrI $\leq$ & ס. זrד & $v \cdot \wedge$ & r, ro & $1 \varepsilon, \ldots$ & 779 & $r, T V$ & $1 \cdot, 0 \mathrm{~V}$ & $0, \Sigma \vee$ & $1 V, \cdot r$ & 171, & $v \cdot 0, \cdot$ & 1०9,. & $1 T, O V$ & $\cdots$ \\
\hline $0 \leq 1 \leq$ & $T \leqslant 70 Y$ & vor & r,T & $1 \varepsilon, 79$ & אדג & $r, 0 \wedge$ & סז,rו & $9, \leqslant 0$ & $1 V, I r$ & $99,$. & $790,$. & $\vee q \varepsilon, \cdot$ & $\mid r, r \wedge$ & .1 \\
\hline 0710 & 70917 & VAO & $Y, \varepsilon)$ & 17,99 & $117 \varepsilon$ & $r, T \leq$ & IV,TY & V,ru & $1 \wedge, \cdot r$ & I & $\wedge r \cdot, T$ & $90 \leqslant,$. & $1 \leqslant, \leqslant 7$ & $r \ldots r$ \\
\hline Vq $\leq \leq$ & TVIT & 109 & $r, \varepsilon q$ & $10, \cdot 1$ & $1 \cdot \leqslant V$ & $r, \wedge q$ & $10,0 \leqslant$ & $\wedge, \wedge 9$ & $r \cdot, \wedge \varepsilon$ & Iro,. & $\Lambda \cdot r, \nu$ & $q r \cdot, \cdot$ & $M, \lambda r$ & $r \ldots r$ \\
\hline $90 V 7$ & $\neg \wedge \neg \leqslant \wedge$ & 170 & $r, \Sigma 0$ & 10,0 & $q \wedge r$ & $r, \wedge r$ & $1 \varepsilon, \Gamma$. & $1 ., 09$ & $r \leq, 01$ & 117, & $\wedge / 9$, & $q r v,$. & $1 \pi, 0$. & $r \ldots \leq$ \\
\hline 997. & $7999 V$ & $\wedge \wedge 9$ & r, & 10,14 & 1.11 & & $1 \leqslant, 0$. & $1 \wedge, \wedge$. & $r 7, \wedge T$ & $19 r$, & $100, \cdot$ & 1.0r,. & $10, \cdot \varepsilon$ & $r \ldots o$ \\
\hline 11 rqr & $V I T \leqslant \Lambda$ & $9 \times 1$ & $r, q$ & $17, \varepsilon \varepsilon$ & 190 & $r, 10$ & $11, r$. & $1 \leq, 07$ & $r q, r v$ & rq^,. & $\wedge \vee q, \cdot$ & $\| \vee \wedge$, & 17,01 & $r \ldots r$ \\
\hline 11911 & VYqE. & $1 \cdots 1$ & $r, r$ & 17,199 & $\wedge \vee q$ & וr, & $I r, I V$ & 17,01 & rY,人T & 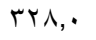 & $91 \vee, \cdot$ & $r \leqslant V,$. & $1 v, 1$. & $r \ldots v$ \\
\hline 10871 & $V \leqslant \leqslant r q$ & 1.71 & r, ro & $10, \leqslant 7$ & NTE & r, Vo & $11, Y \varepsilon$ & $r \varepsilon, r$ & & "ו,. & $971, \cdot$ & $r \cdot 1, \cdot$ & $\mid \vee, \varepsilon \wedge$ & $r \ldots \Lambda$ \\
\hline $1 \vee 9 \wedge \wedge$ & 87.99 & 1.94 & ץ, \^ & $|7,7|$ & $\wedge \vee \wedge$ & $r, q r$ & 11,10 & $r \cdot, \Sigma V$ & $\varepsilon \cdot, \varepsilon \wedge$ & $r V \cdot, \cdot$ & $9 \wedge 1, \cdot$ & 1 rol, & $17, \varepsilon \varepsilon$ & $r \ldots q$ \\
\hline IATIY & $\vee \vee \wedge \varepsilon$. & $1 \pi .0$ & $\varepsilon, \Gamma \wedge$ & $19, \cdot r$ & $9 \leq 9$ & r, & $1 r, 00$ & $\Gamma \cdot, \Sigma \varepsilon$ & or,vr & 190, & १९५, & isr,. & $10, Y$. & $r+1 \cdot$ \\
\hline rIYVT & V9ד11 & & $\varepsilon, \mid \wedge$ & $19,1 \mathrm{~V}$ & $\cdots$ & דr, & $14,9 \leq$ & $r \cdot, \Sigma r$ & $\circ \wedge, 7$. & $r \mid r$, & १^^, • & Ir.r., & 10,11 & $r .11$ \\
\hline$r \ldots \leqslant \Lambda$ & N107V & ITVT & זד, ז & 19,71 & $1 \cdot T V$ & $r, \cdot 1$ & Ir, IV & rr, $\cdot q$ & $7 r, 9$. & $r \cdot \Lambda, \cdot$ & $99 \cdot, \cdot$ & 1100, & $1 \leqslant, 17$ & $r .1 r$ \\
\hline$r .7 \leq \Lambda$ & קדצטא & $1 \leq 0 \leq$ & r., & $19,0 \leq$ & $111 \mathrm{~V}$ & $r, 7 q$ & $10, \cdot v$ & $r r, \Sigma 0$ & $7 V, 10$ & $r \cdot v, \cdot$ & $970,$. & IrqA,. & 10,01 & $r \cdot 1 r$ \\
\hline
\end{tabular}

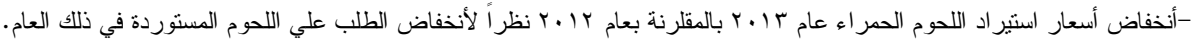

المصدر: جمعت وحسبت من بيانات الجهاز المركزي للتعبئة العامة والإحصاء، نشرة استنهالك السلع، الكتاب الإحصائي

السنوي، أعداد مختلفة.

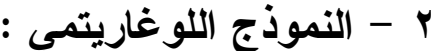

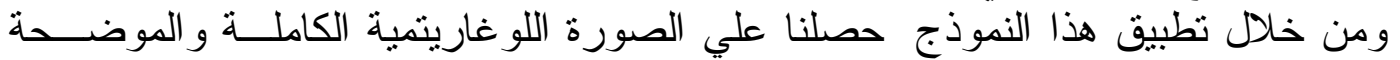

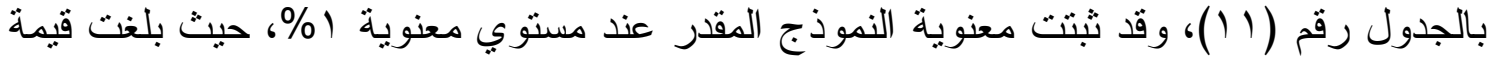

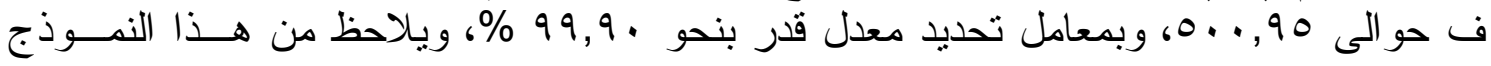

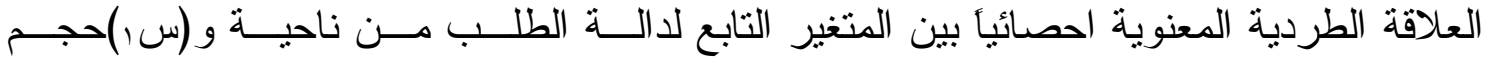

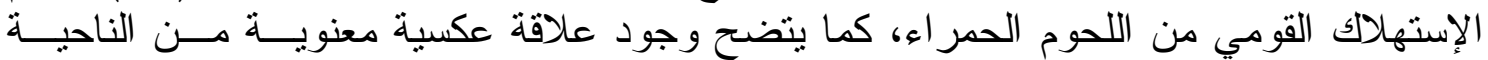

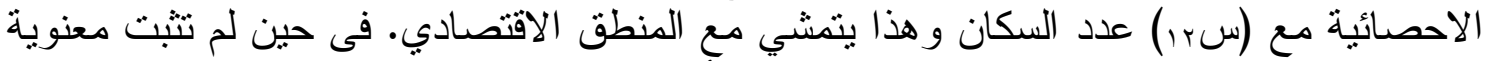

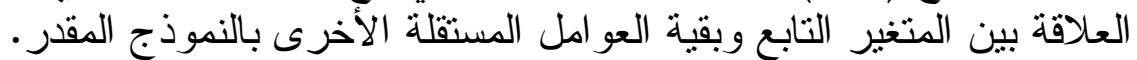

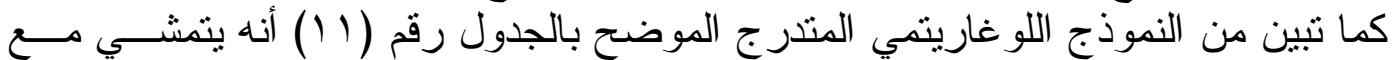

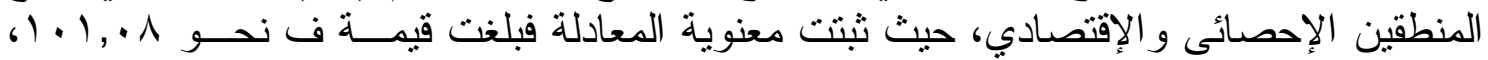

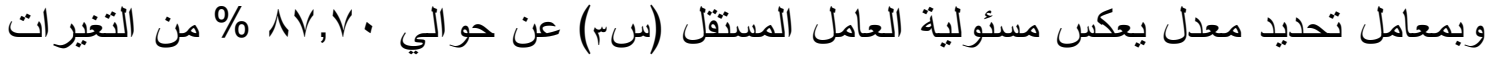

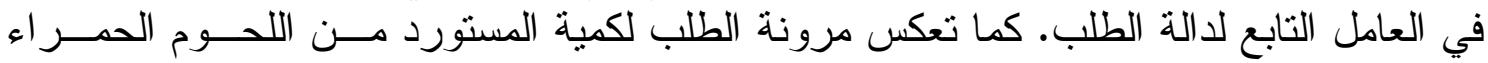

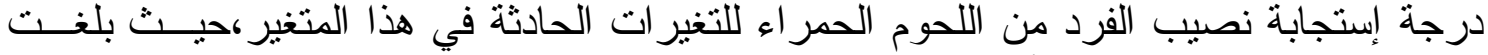

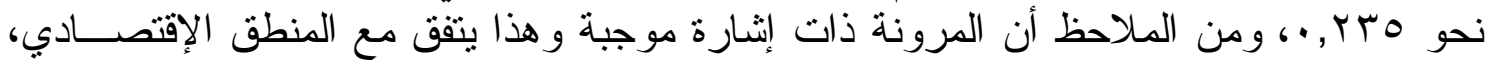

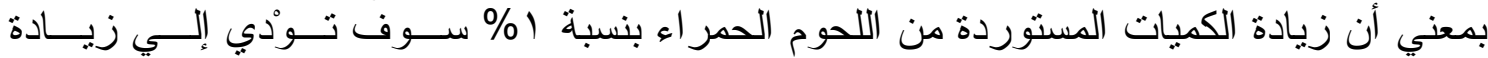
نصيب الفرد من اللحوم الحمر اء المحلية بنسبة هبr بر , •\% فى السنة. 
جدول رقم 1 ـ مصفوفة الارتباط بين العامل التابع (نصيب الفرد مــن اللحسـوم الحمــراء) و العوامـلـ

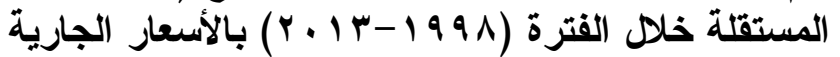

\begin{tabular}{|c|c|c|c|c|c|c|c|c|c|c|c|c|c|c|}
\hline $\mathbf{X}_{13}$ & $X_{12}$ & $\mathbf{X}_{11}$ & $X_{10}$ & $X_{9}$ & $\mathbf{X}_{8}$ & $\mathbf{X}_{7}$ & $\mathbf{X}_{6}$ & $\mathbf{X}_{5}$ & $\mathbf{X}_{4}$ & $\mathbf{X}_{3}$ & $\mathbf{X}_{2}$ & $\mathbf{X}_{1}$ & $\mathbf{Y}$ & \\
\hline & & & & & & & & & & & & & 1 & $\mathbf{Y}$ \\
\hline & & & & & & & & & & & & $T$ & $* *, 9,4$ & $\mathbf{X}_{1}$ \\
\hline & & & & & & & & & & & 1 & ***, q $\{Y$ & ***,, $\mathrm{V} \cup \mathrm{V}$ & $\mathbf{X}_{2}$ \\
\hline & & & & & & & & & & 1 & 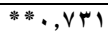 & ***,q१ए & ***, , q40 & $\mathbf{X}_{3}$ \\
\hline & & & & & & & & & 1 & $* *$, , प9 & $* *, \vee \vee \wedge 0$ & ***, $7 \leqslant 9$ & $\cdot, Y \wedge O^{\prime}$ & $\mathbf{X}_{4}$ \\
\hline & & & & & & & & 1 & ${ }^{* *} \cdot, \wedge \varepsilon \varepsilon$ & ".,०१Y & $* *, 9 Y 0$ & $\mid * *, \Lambda Y V$ & ".,०VA & $\mathbf{X}_{5}$ \\
\hline & & & & & & & $T$ &., $.4 \mathrm{VV}$ & $\cdot, 1 \leqslant 0$ & $\cdot, \cdot \wedge \varepsilon-$ & $\cdot, Y O \xi$ & ., $1 \times 4$ & $\cdot, \ldots 4$ & $\mathbf{X}_{6}$ \\
\hline & & & & & & $T$ & .,.74- & $* *, 010$ & $\cdot, \mathrm{rAV}$ & . & "*,० ४ \& & $\cdot, \varepsilon \vee \wedge$ & . & $\mathbf{X}_{7}$ \\
\hline & & & & & $T$ &., $11 \mathrm{~V}$ & 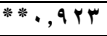 & $\cdot, \leqslant 17$ & $\cdot, \varepsilon \mathrm{VV}$ &., $19 \mathrm{~V}$ & $*, \wedge 0$. &.,$\{01$ & $\cdot, Y r V$ & $\mathbf{X}_{8}$ \\
\hline & & & & $T$ & ***, & $\cdot, \varepsilon \vee \mathrm{V}^{2}$ & $\cdot, r \cdot 0$ & $* *, \wedge \circ 4$ & $* * *, q \cdot \Lambda$ & ***,० & $* * \cdot, \wedge \vee \wedge$ & **, & $\cdot, \varepsilon \vee 1$ & $\mathbf{X}_{9}$ \\
\hline & & & 1 & $* *, \wedge \leqslant 9$ & $\cdot, \mathrm{rVV}$ & **, * T V V & $\cdot, \cdot, \leqslant 4$ & ***,90Y & ***,人ץะ & $* \cdot, 0 \vee \leqslant$ & $* * ., 9.0$ & ***,A।I & $* \cdot, 091$ & $X_{10}$ \\
\hline & & 1 & $* *, \wedge \vee \uparrow$ & $* *, 9 \vee 4$ & *.,Tr. & $\cdot, \varepsilon, 4$ & - Y Y & $* *, \wedge ৭ \wedge$ & ***, *TY & $* \cdot, \because \mathrm{V}$ & $* *, 9,9 \%$ & ***,人 & *., Oro & $X_{11}$ \\
\hline & 1 & $* *, 99$. & $* *, \wedge 40$ & $* *, 901$ & ${ }^{*} \cdot, \mathrm{Y} \cdot \mathrm{V}$ & ., \&ाY & $\cdot, \mathrm{r} O \mathrm{~V}$ & $* *, 9) \leqslant$ & $* *, 9 \ldots$ & $* *, \uparrow \wedge$. & $* *, 9 \leqslant \Gamma$ & $* * *, \wedge \wedge \varepsilon$ & $*, ., 9 \wedge$ & $\mathrm{X}_{12}$ \\
\hline 1 & $* *, 94$ & $* *, q \leqslant V$ & $* *, \vee \vee 0$ & $* \cdots, q, q$ & "*,OYV &.,$\times 79$ & $\cdot, 1 \times 0$ & $* *, \wedge \%$ & "* *,QYT & $\because \cdot,, 0 \wedge \varepsilon$ & $* *, \wedge \uparrow q$ & **., $\vee \vee V I$ & $\cdot, \varepsilon r v$ & $\mathbf{X}_{13}$ \\
\hline
\end{tabular}

المصدر : حسبت من بيانات الجدول رقم (9) السابق •

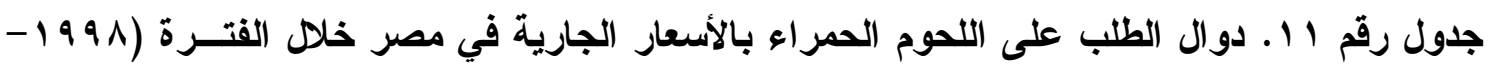

\begin{tabular}{|c|c|c|c|}
\hline ju & ف & المعادلة & البيان \\
\hline$\cdot, 999$. & $1 \leq V \cdot, 74$ & 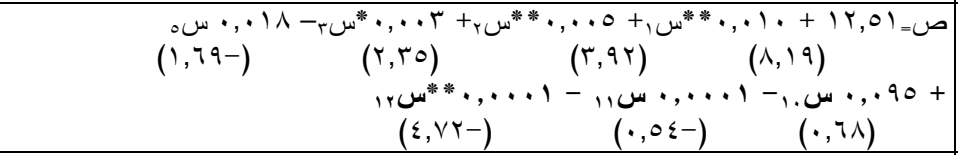 & خطى كامل \\
\hline$\cdot, \wedge \neg \varepsilon$. & $97,7$. & 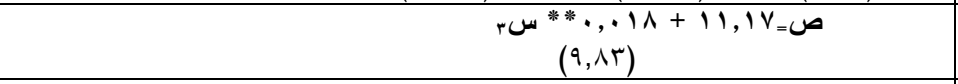 & خطى مرحلى \\
\hline., 999. & $0 \cdots, 90$ & 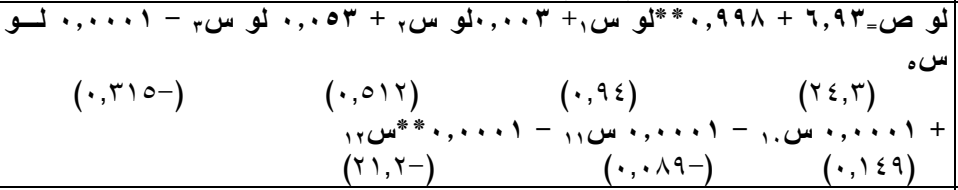 & لوغاريتمى كامل \\
\hline$\cdot, \wedge \vee \vee \cdot$ & $1 \cdot 1,1$ & 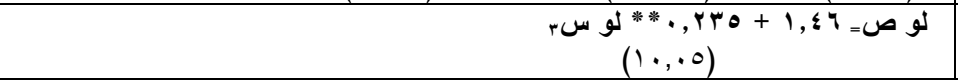 & لوغاريتمي مرحلى \\
\hline
\end{tabular}

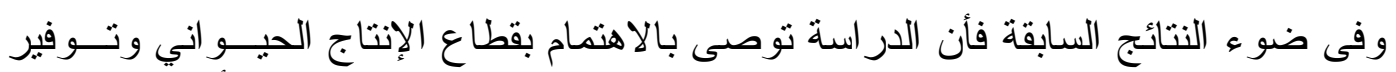

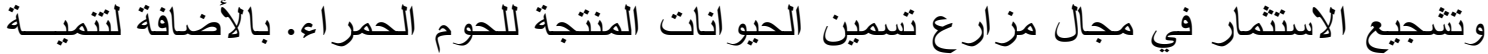

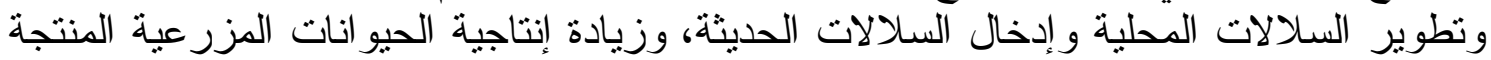

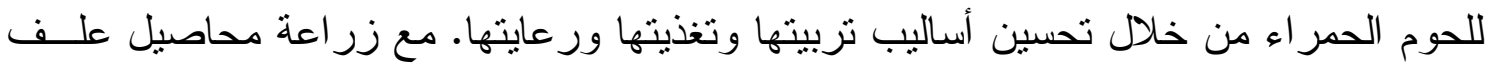

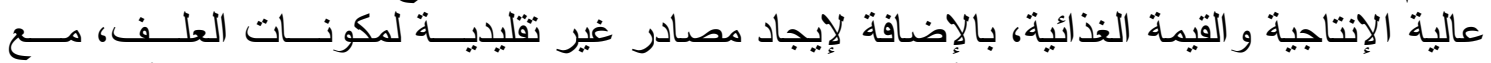

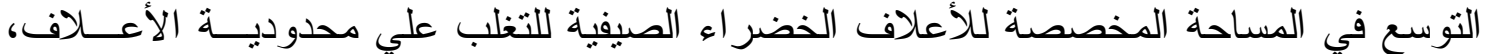

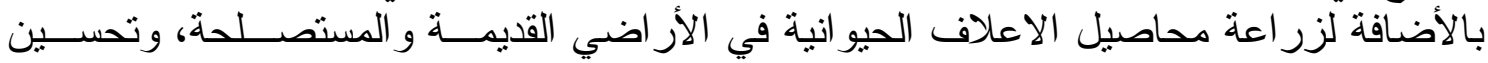

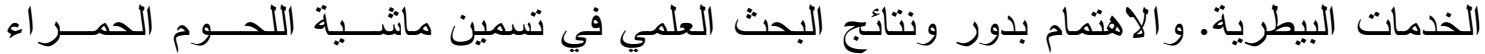

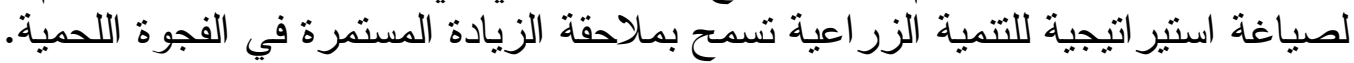
الملخص

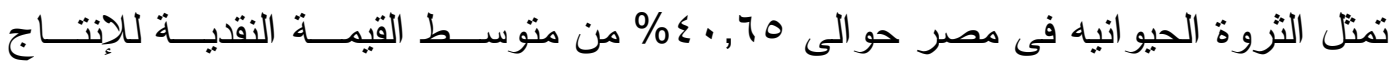

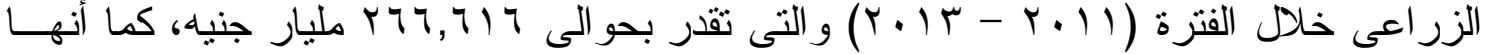

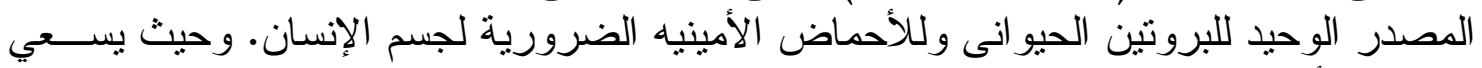

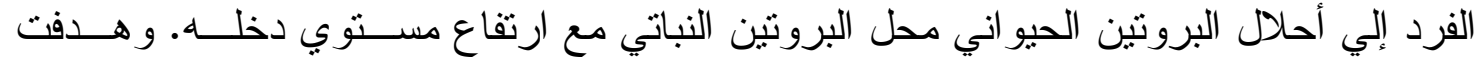

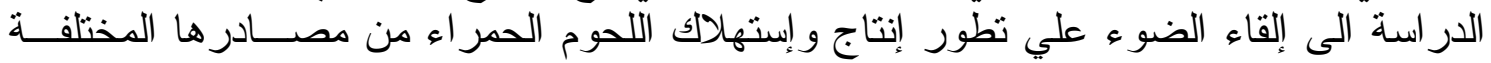




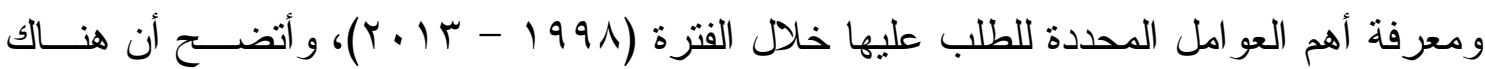

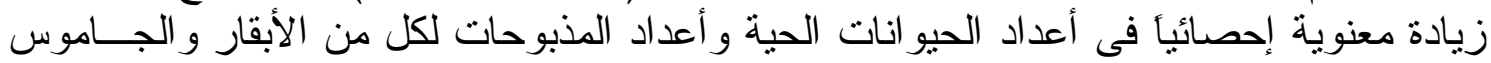

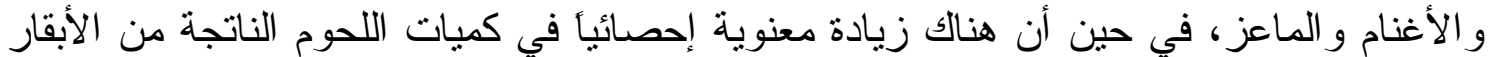

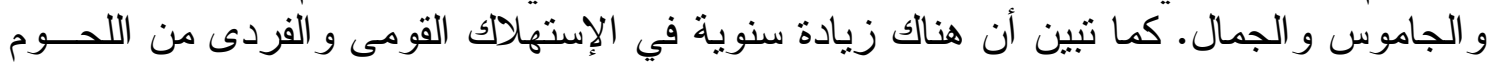

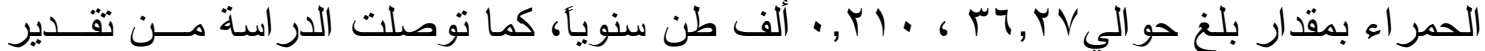

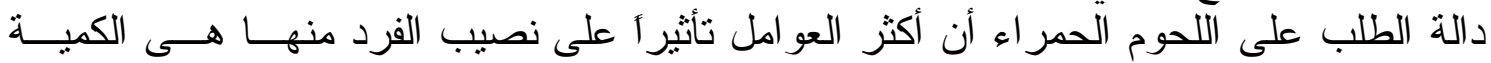

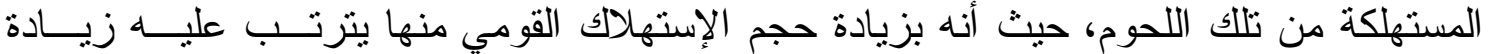

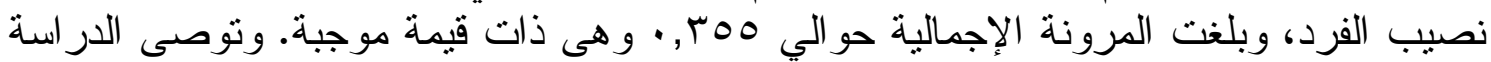

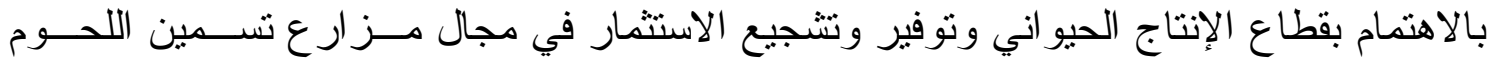

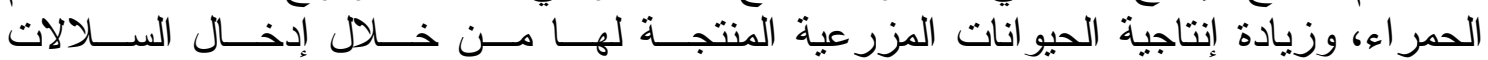
الحديثةو تحسين أساليب تربيتها وتغذيتها ور عايتها.

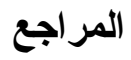

1- الجهاز المركزي للتعبئة العامة و الإحصاء،نشرة تقلير اتا لدخل مــن القطــــاع الزر اعي،أعـداد

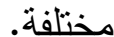

r- الجهاز المركزي للتعبئة العامة و الإحصاء ، النثرة السنوية لإحصاءات الثروة الحيو انية،الكتاب الإحصائي السنوي، أعداد مختلفة.

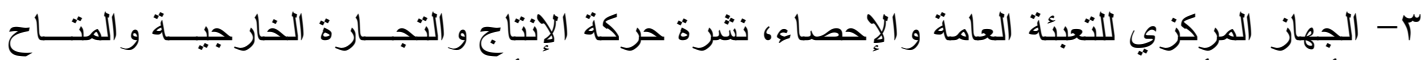

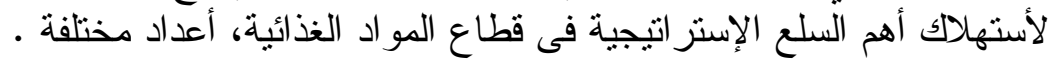

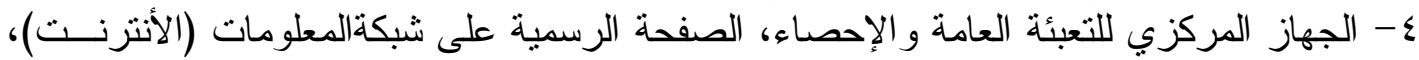
URI: WWW.Capmas.gov.eg 0- الجهاز المركزي للتعبئة العامة و الإحصاء، المكتبة الإلكثرونية المركزية، القاهرة .

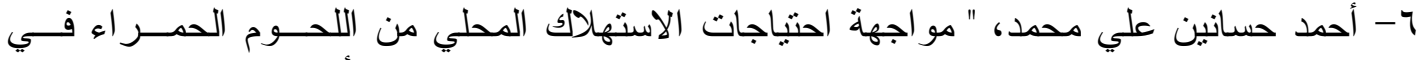

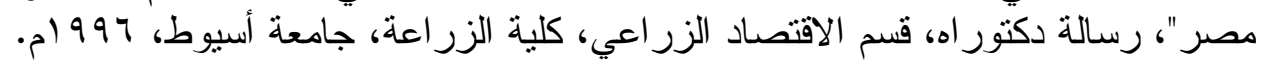

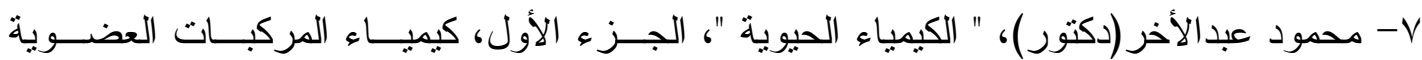

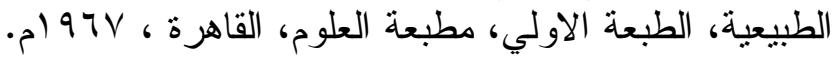




\title{
The Current and Future Position of the Production and Consumption of Red Meat and its Demand in Egypt \\ Ahmed M. Zedan ${ }^{2}$; A. Ibrahim Mohamed ${ }^{1}$; Suzan A. Aboelmajd ${ }^{1}$ and Mndouh E. Mahmoud ${ }^{2}$ \\ ${ }^{1}$ Department of Agricultural Economics, Faculty of Agriculture, Assiut University. ${ }^{2}$ Agriculture Research Center
}

\begin{abstract}
Representing livestock in Egypt, $40.65 \%$ of the average monetary value of agricultural production during (2011-2013), amounting to approximately 266616 million pounds, and it is consider tend to the only source of animal protein and amino acids essential to the human body. The individualtend to bring replace animal protein vegetable protein with a high level of income. The study aimed to shed light on the evolution of the production and consumption of red meat from different sources and the most important determinants of demand during (1998 2013).
\end{abstract}

It turned out that there is a statistically significant increase in the number of live animals and numbers slains each of the cows, buffalo, sheep and goats, while there is a statistically significant increase in the quantities of meat derived from cattle and buffalo and camels. As it turns out that there is an annual increase in both ofindividualnational consumption by about $36.27,0.210$ thousand tons per year respectively, The study also shows by that estimating the demandfunction of red meat is that the main factor is the amount of imports of red meat, as it increased the amount of imports of red meat results in increased per capita and that the relationship that bound together are a direct correlation, And the total flexibility of these factors combined with about 0.235 a positive value .

The study recommends the attention of animal production and providing sector and encourages investment in the field of red meat fattening farms; increasing farm animals have produced productivity through the introduction of modern breeds and improving bred and nurtured methods. 